\title{
Improvement of the sound absorption of flexible micro-perforated panels by local resonances
}

\author{
S. W. Ren ${ }^{1,3,4,5^{*}}$, L. Van Belle ${ }^{3,6}$, C. Claeys ${ }^{3,6}$, F. X. Xin ${ }^{4,5 *}$, T. J. Lu $^{2,4,5^{*}}$, \\ E. Deckers ${ }^{3,6}$, W. Desmet ${ }^{3,6}$ \\ ${ }^{I}$ School of Marine Science and Technology, \\ Northwestern Polytechnical University, Xi'an 710072, PR China \\ ${ }_{2}^{2}$ State Key Laboratory of Mechanics and Control of Mechanical Structures, \\ Nanjing University of Aeronautics and Astronautics, Nanjing 210016, PR China \\ ${ }^{3}$ Department of Mechanical Engineering, KU Leuven \\ Celestijnenlaan 300 B, B-3001 Heverlee (Leuven), Belgium \\ ${ }^{4}$ MOE Key Laboratory for Multifunctional Materials and Structures, \\ Xi'an Jiaotong University, Xi'an 710049, PR China \\ ${ }^{5}$ State Key Laboratory for Mechanical Structure Strength and Vibration, \\ Xi'an Jiaotong University, Xi'an 710049, PR China \\ ${ }^{6}$ DMMS lab, Flanders Make, Belgium
}

To further improve the sound absorption enhancement of flexible micro-perforated panels (FMPPs), a new sort of perforated sound absorbers - metamaterial-based micro-perforated panels (MMPPs) - is proposed by combining a micro-perforated host panel and local resonators (LRs) attached on a sub-wavelength scale, targeting the flexural waves. Theoretical and numerical models show that MMPPs are able to further enhance sound absorption in a wide frequency range. The theoretical model is developed based on the effective medium method as the structural wavelength in the host panel is much larger than the distance between the LRs, and the full simulation model, including visco-thermal effects, is conducted by utilizing multi-physical coupling integrated in COMSOL. Besides, a structural finite element unit cell method is used to evaluate the stop band behavior of the MMPP. Good agreement is achieved between the theoretically predicted acoustical properties and the simulation results for both conventional FMPPs and the proposed MMPPs, validating the numerical and theoretical models. Both models reveal that the sound absorption enhancement of the MMPP stems from the resulting acoustic surface impedance improvement, caused by the sub-wavelength attached local resonances. The effect of key properties of the LRs (i.e. mass, damping and multiple resonances) on the sound absorption performance of

*Corresponding author: shuwei.ren@ hotmail.com (S. W. Ren) or fengxian.xin@gmail.com (F.X. Xin) or tjlu@mail.xjtu.edu.cn (T.J. Lu). 
MMPPs is then analyzed by applying the theoretical model and effective frequency-adjustability of the absorption enhancement performance is found. The proposed MMPP shows great potential for the noise reduction industry.

Keywords: metamaterial; micro-perforated panel; absorption improvement; stop band; effective medium method 


\section{Introduction}

As artificial materials with distinctive microscopic structures, acoustic metamaterials $^{[1-3]}$ possess a range of peculiar properties, such as negative mass density $^{[4,5]}$, negative modulus ${ }^{[6]}$, negative refraction index ${ }^{[7]}$, etc. These characteristics prompt a wide spectrum of novel engineering applications, including acoustic cloaking $^{[8,9]}$, power harvesting ${ }^{[10]}$ and acoustic imaging ${ }^{[11]}$. The application areas of acoustic metamaterials are still being further explored and expanded.

Recently, sound absorption improvement, regarded as a conventional yet challenging issue in numerous applications, through utilizing metamaterial concepts attracts increasing attention. Among existing works, viscous-thermal dissipation ${ }^{[12-16]}$ has been adopted as the key mechanism underlying the sound energy absorption. In particular, the strategy of space-coiling ${ }^{[17]}$ significantly condenses the thickness of materials to achieve total sound absorption $^{[18]}$ and the smallest thickness-to-wavelength ratio can even reach $\sim 1 / 223^{[19]}$. However, the induced side effect - large acoustic reactance mismatch - shrinks the functional frequency range to a very narrow band. Extraordinary absorbers ${ }^{[20,21]}$ further developed from conventional porous structures/materials exhibit magnificent sound absorption performance in a broad frequency range, but their thickness-to-wavelength ratios are typically rather large. Upon manipulating the acoustical anisotropy of the metamaterial-composed impedance-matching shells ${ }^{[22,23]}$ omni-directionally impinging sound waves can be bent into an internal dissipative core and hence be absorbed effectively, but large material volumes are still needed. Besides viscous-thermal dissipation, alternative sound absorption mechanisms have also been explored, such as coherent control ${ }^{[24,25]}$ of sound waves, multiple resonance ${ }^{[26]}$, and critical coupling $^{[27]}$. Nonetheless, at present, an applicable balance between the width of the functional frequency range and the limited allowable material/structure thickness is not yet achieved using the abovementioned approaches.

Among the most widely used sound absorbing materials/structures are micro-perforated panels (MPPs) ${ }^{[28]}$. These have been optimized from different perspectives ${ }^{[29-32]}$ to dissipate sound energy in lower and/or broader frequency ranges. It has been shown that the flexibility ${ }^{[29,30]}$ of MPPs leads to overall panel vibrations, which can cause extraordinary absorption peaks before those achieved by conventional panels. However, these additional peaks are always followed by 
undesired dips, which also stem from the overall panel vibrations. More specifically, "the magnitude and phase of the air-frame relative velocity over the MPP determines the beneficial or detrimental effect of these resonances" ${ }^{\text {[33] }}$. In literature, adding local resonators (LRs) to bare panels is an effective way to prevent undesired panel vibrations. Metamaterial panels ${ }^{[34-37]}$ have shown potential for enhanced sound insulation relative to conventional bare panels, at least in targeted frequency zones, referred to as stop bands. Inspired by this concept, as well as the work of Ruiz et al ${ }^{[14]}$ to combine micro-slits of sub-millimeter dimensions with a metamaterial panel, this work introduces a new sort of MPP, the metamaterial-based MPP (MMPP), by attaching structural local resonances to a conventional flexible MPP (FMPP). Mass-spring-damper resonators are attached to one face of an FMPP, targeting the acoustically relevant flexural waves, to further enhance their sound absorption ability, especially in the frequency range where the FMPP cannot work well due to overall panel vibration. The spacing of the resonators is sub-wavelength compared to the wavelength of the flexural waves in the FMPP, according to the rules of local resonant metamaterial design ${ }^{[35]}$. The applied enhancement strategy is as follows. Firstly, the overall panel vibration of an FMPP is utilized to enhance sound absorption in the frequency range below the pore-cavity peak of a rigid MPP. Secondly, the stop-band behavior of a metamaterial panel is exploited to impair the overall panel vibrations in a specific frequency range to avoid undesired absorption dips. It will be shown that the introduced local resonances not only lift the undesired dips but also produce superior peaks compared to a rigid MPP having the identical perforation diameter and perforation ratio. Consequently, the MMPP is demonstrated as a further step towards a practical balance between a broad operating frequency range and a low structure thickness.

This work is organized as follows. In Section 2, the proposed MMPP is defined. Next, the theoretical modeling based on the effective medium method is described in Section 3. In Section 4, a full acoustic-structural simulation model is established to validate the theoretical model as well as to reveal the physical mechanism underlying the absorption enhancement. A finite element unit cell method to extract the stop band behavior is briefly explained in Section 5. The influence of the key properties of LRs on the MMPP performance is investigated in Section 6. Finally, the main conclusions are given in Section 7. 


\section{Metamaterial-based micro-perforated panels}

The objective of combining FMPPs with locally resonant metamaterial behavior is to further improve the sound absorption performance of FMPPs. It has been proven that the FMPPs, via panel-cavity resonances, are able to enhance the sound absorption of rigid MPPs in the lower frequency range $\mathrm{e}^{[30]}$, when the fundamental frequency of FMPP is proposed to coincide with that corresponding to the pore-cavity peak of a rigid MPP. In the present study, the attached LRs are designed to improve the undesired absorption dips due to overall vibrations that follow the enhanced peaks.

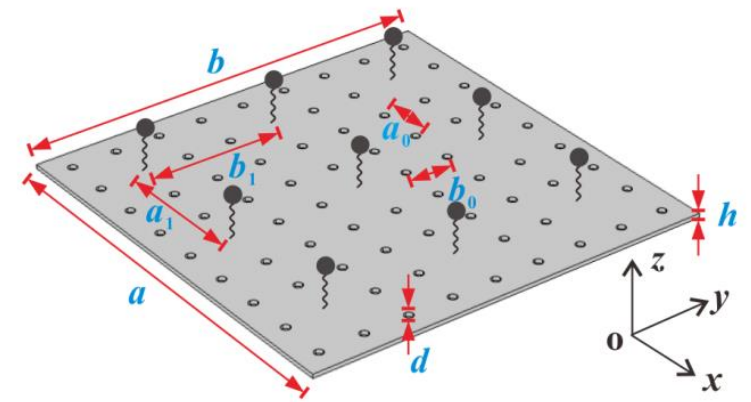

Fig. 1. (Color online) Schematic representation of the proposed MMPP.

Table 1 Topological and material parameters of the reference MMPP.

\begin{tabular}{cccc}
\hline \hline Component & Name & Symbol & Value \\
\hline \multirow{2}{*}{ Length } & $a$ & $63.5 \mathrm{~mm}$ \\
Width & $b$ & $63.5 \mathrm{~mm}$ \\
Thickness & $h$ & $0.5 \mathrm{~mm}$ \\
host panel & Perforation diameter & $d$ & $0.8 \mathrm{~mm}$ \\
& Center-to-center distance & $a_{0} / b_{0}$ & $7.9 \mathrm{~mm}$ \\
& Young's modulus & $E_{\mathrm{p}}$ & $69 \mathrm{GPa}$ \\
& Density & $\rho_{\mathrm{p}}$ & $2730 \mathrm{~kg} / \mathrm{m}^{3}$ \\
& Poisson's ratio & $v_{\mathrm{p}}$ & 0.33 \\
& Damping loss factor & $\eta_{\mathrm{p}}$ & $0.5 \%$ \\
& Resonator mass & $m_{\mathrm{r}}$ & $34.4 \times 10^{-6} \mathrm{~kg}$ \\
& Spring constant & $k_{\mathrm{r}}$ & $487.13 \mathrm{~N} / \mathrm{m}$ \\
& Damping loss factor & $\eta_{\mathrm{r}}$ & $2 \%$ \\
& Amount & $N$ & 16 \\
\hline \hline
\end{tabular}

The proposed MMPP is composed of a micro-perforated host panel combined with a number of local resonators (LRs), which are attached to one face of the host panel, as depicted in Fig. 1. The considered host panel has a rectangular shape with 
length $a$, width $b$ and thickness $h$. The micro-perforations in the host panel are circular with diameter $d$ and the center-to-center distance between the micro-perforations is $a_{0}$ and $b_{0}$, along the length- and width-direction respectively. Since this paper proposes a conceptual idea to utilize stop band behavior to enhance sound absorption, idealized mass-spring-damper resonators ${ }^{[34,35]}$ are adopted as LRs, consisting of a point-mass $m_{\mathrm{r}}$ and a spring with complex spring constant $k_{\mathrm{r}}\left(1+i \eta_{\mathrm{r}}\right)$ to take into account the damping through a loss factor $\eta_{\mathrm{r}}$. In literature, some state-of-art resonators ${ }^{[14,38]}$ (e.g. the mass-cantilever resonant structure ${ }^{[38]}$ ) have been proven capable of resulting in stop bands for metamaterial-panels, which are potential realizations of the mass-spring-damper resonators. For the single-degree-of-freedom mass-spring-damper resonators, the damping loss factor $\eta_{\mathrm{r}}$ is the most simplified form of the structural damping, which has been validated as a simple but realizable way to include damping in real $\operatorname{LRs}^{[38]}$. Some other more complicated but more accurate forms of damping, such as viscous and visco-elastic damping, are not included here. Moreover, in following theoretical and numerical studies the volumes of the resonators are assumed to be 0 to ignore the influence of the LR movements on the sound field in the back cavity, and the interactions between the resonators and the host panel are considered through point-loads only along $z$ direction (i.e. ignoring the swing movement). These assumptions may limit the general applicability of the deduced results in this paper for guiding further experiments and practical applications. However, when the mass of a mass-cantilever structure has the same density with the host panel, the total volume of all the resonators will not exceed $1 \%$ of the volume of the back cavity. Meanwhile, considering the sub-wavelength requirement, point-load interactions are a reasonable simplification. The LRs are separated with distance $a_{1}$ and $b_{1}$ along the length- and width-direction, respectively. Table 1 lists the geometrical and material parameters of the reference MMPP studied numerically and theoretically in this paper.

The material considered for the host panel is aluminum. The in-plane dimensions of the host panel, $63.5 \mathrm{~mm} \times 63.5 \mathrm{~mm}$, are designed in view of the standard ${ }^{[39]}$ for well-developed sample testing systems (e.g. B\&K 4206 impedance tube). The remaining geometrical parameters are designed mainly to demonstrate the sound absorption enhancement capability of an MMPP in the frequency range of interest 
(100-1000 Hz), considering the limitations of existing computational resources for numerical simulations. Consequently, the resulting perforation ratio $\phi$ is $0.8 \%$. For a backing cavity of depth $D=48 \mathrm{~mm}$, the pore-cavity peak of a rigid MPP having the same perforation diameter and perforation ratio as the proposed MMPP is located at $599.18 \mathrm{~Hz}$. To introduce locally resonant metamaterial behavior, 16 identical LRs tuned to $598.91 \mathrm{~Hz}$ (the same as the fundamental frequency of the host panel) are attached to the host panel with a regular spacing of $15.9 \mathrm{~mm} \times 15.9 \mathrm{~mm}$ to satisfy the sub-wavelength criterion. This results in a relative mass addition of $10 \%$. It will be seen in the following that only $(1,1)$ modes exist for the proposed panel size in the frequency range 100-1000 Hz. Although a single mass-spring-damper resonator would suffice to prevent the targeted panel vibration for the considered panel size, designing it as a locally resonant metamaterial will install global vibration reduction over the entire panel irrespective of the panel size, and will reject all panel modes in the targeted stop band as to restore the sound absorption behavior.

In what follows it will be demonstrated that the proposed MMPP allows higher sound absorption in a wider frequency range, with a practical balance between a broad working range and a low structure thickness (including the backing cavity).

\section{Theoretical modeling based on effective medium method for sound} absorption

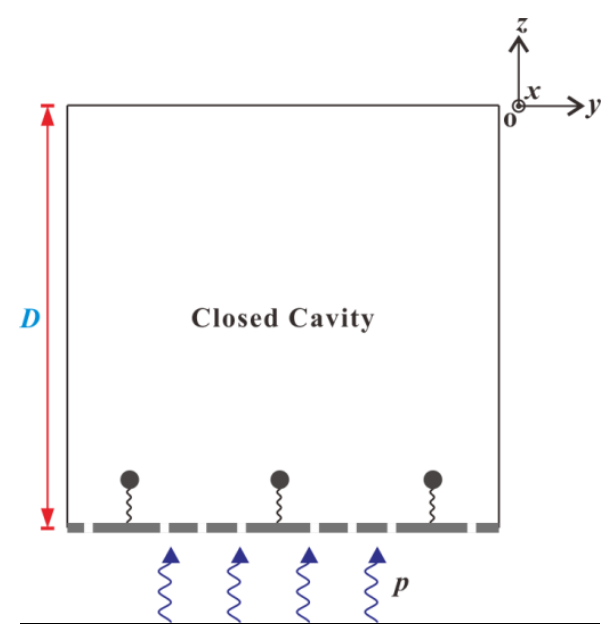

Fig. 2. (Color online) Side view of a simply supported MMPP backed by a closed rectangular cavity, with sound wave normally impinging on the lower face of the MMPP.

In this section a theoretical model is developed to predict the sound absorption performance of the proposed MMPP. For an MMPP backed by a closed rectangular cavity surrounded with rigid walls, the problem setting can be presented as in Fig. 2 . 
The four edges of the MMPP are simply supported, and a plane sound wave is impinging normally on the lower face of the MMPP.

The procedure provided by Lee et al. ${ }^{[29]}$ is first introduced to describe the considered vibroacoustic problem of Fig. 2. In particular, the acoustic field inside the closed cavity is modeled via its acoustic velocity potential $\Phi$, which is governed by the homogeneous wave equation ${ }^{[40]}$, as:

$$
\nabla^{2} \Phi-\frac{1}{c_{0}^{2}} \frac{\partial^{2} \Phi}{\partial t^{2}}=0
$$

where $c_{0}=343 \mathrm{~m} / \mathrm{s}$ is the sound speed in the ambient environment. Corresponding to the problem of Fig. 2, the normal air particle velocity at the surrounding walls is equal to zero except at the upper face of the MMPP, where the particle velocity equals the distributed velocity $v_{\mathrm{D}}(x, y, t)$ of the upper face of the MMPP or of the air particle velocity inside the perforations of the MMPP, due to velocity continuity ${ }^{[41-44]}$. This results in following boundary conditions:

$$
\begin{array}{ll}
x=0, a: & \frac{\partial \Phi}{\partial x}=0 \\
y=0,-b: & \frac{\partial \Phi}{\partial y}=0 \\
z=0: & \frac{\partial \Phi}{\partial z}=0 \\
z=-D: & \frac{\partial \Phi}{\partial z}=v_{\mathrm{D}}(x, y, t)
\end{array}
$$

As abovementioned, the dimensions and the shapes of LRs are ignored and LRs are modelled as point force interactions with the plate, thus the upper face of the MMPP is regarded as a flat surface here.

In theoretical modelling, velocity and pressure averaging are frequently used, which significantly simplifies the modeling procedure, but still ensures sufficient accuracy of the predicted results ${ }^{[29]}$. This will be proven in the validation section (i.e. Section 6.1). From a physical point of view, the relative velocity of air particles inside the perforations compared to that of the panel frame determines the viscous dissipation occurring inside the boundary layers adjacent to the perforation walls. The relation between the average air particle velocity inside the perforations $\bar{v}_{\mathrm{o}}$, the average panel frame velocity $\bar{v}$, and the average pressure drop $\Delta \bar{p}$ through the perforations is given approximately by: 


$$
\operatorname{Re}\left(Z_{\mathrm{o}}\right)\left(\bar{v}_{\mathrm{o}}-\bar{v}\right)+\mathrm{i} \operatorname{Im}\left(Z_{\mathrm{o}}\right) \bar{v}_{\mathrm{o}}=\Delta \bar{p}
$$

in which $Z_{\mathrm{o}}$ is the acoustic impedance of the perforations. Here, the approximate model proposed by $\mathrm{Maa}^{[28]}$ for $Z_{\mathrm{o}}$ is adopted and its real (acoustic resistance) and imaginary (acoustic reactance) part are respectively given by:

$$
\begin{aligned}
& \operatorname{Re}\left(Z_{\mathrm{o}}\right)=\frac{32 \eta h}{d^{2}}\left(\sqrt{1+\frac{k^{2}}{32}}+\frac{\sqrt{2}}{8} k \frac{d}{h}\right) \\
& \operatorname{Im}\left(Z_{\mathrm{o}}\right)=\rho_{0} \omega h\left(1+1 / \sqrt{9+\frac{k^{2}}{2}}+0.85 \frac{d}{h}\right)
\end{aligned}
$$

where $d$ is the perforation diameter, $h$ is the panel thickness, $\eta=1.95 \times 10^{-5} \mathrm{~Pa} \cdot \mathrm{s}$ is the dynamic viscosity of air, $\rho_{0}=1.23 \mathrm{~kg} / \mathrm{m}^{3}$ is the static air density, $\omega$ is the angular frequency, and $k=d \sqrt{\omega \rho_{0} / 4 \eta}$ is proportional to the ratio of perforation radius to viscous boundary layer thickness inside the perforations.

The three averaging operations in Eq. (3) are calculated as:

$$
\begin{aligned}
& \Delta \bar{p}=\frac{\int_{0}^{b} \int_{0}^{a}\left(p-p_{\mathrm{D}}\right) \mathrm{d} x \mathrm{~d} y}{a b}=p-\bar{p}_{\mathrm{D}} \\
& \bar{v}=\frac{\int_{0}^{b} \int_{0}^{a} v \mathrm{~d} x \mathrm{~d} y}{a b} \\
& \bar{v}_{\mathrm{o}}=\frac{\int_{0}^{b} \int_{0}^{a} v_{\mathrm{o}} \mathrm{d} x \mathrm{~d} y}{a b}
\end{aligned}
$$

in which $p$ is the uniformly distributed sound pressure at the lower face of the MMPP and $p_{\mathrm{D}}$ is the distributed pressure at its upper face. Alternatively $\bar{v}$ can also be determined through the impedance $Z$ of the panel frame of the MMPP as:

$$
\bar{v}=\frac{\Delta \bar{p}}{Z}
$$

The averaged velocity $\bar{v}_{\mathrm{D}}$ at the upper face may be calculated through

$$
\bar{v}_{\mathrm{D}}=(1-\phi) \bar{v}+\phi \bar{v}_{\mathrm{o}},
$$

where $\phi$ is the perforation ratio of the MMPP.

The transfer impedance of the entire proposed MMPP system may be defined as:

$$
Z_{\mathrm{t}}=\frac{\Delta \bar{p}}{\rho_{0} c_{0} \bar{v}_{\mathrm{D}}}
$$


which is normalized by the characteristic impedance of air $Z_{\mathrm{c}}=\rho_{0} c_{0}$. Subsequently, the surface impedance of the MMPP is approximated ${ }^{[29]}$ for $\phi<<1$ as:

$$
Z_{\mathrm{s}}=\frac{p}{Z_{\mathrm{c}} \bar{v}_{\mathrm{D}}} \approx \frac{\bar{Z}_{\mathrm{o}} Z}{Z_{\mathrm{c}}\left(\bar{Z}_{\mathrm{o}}+Z\right)}-\mathrm{i} \cot \left(\frac{\omega D}{c_{0}}\right),
$$

in which $\bar{Z}_{\mathrm{o}}=Z_{\mathrm{o}} / Z_{\mathrm{c}} \phi$ is the overall normalized impedance of a rigid MPP and $D$ is the depth of the backing cavity. Hence, the sound absorption coefficient $\alpha$ of the system can be obtained as:

$$
\alpha=\frac{4 \operatorname{Re}\left(Z_{\mathrm{s}}\right)}{\left[1+\operatorname{Re}\left(Z_{\mathrm{s}}\right)\right]^{2}+\operatorname{Im}\left(Z_{\mathrm{s}}\right)^{2}} .
$$

Next, the present work further elaborates the theoretical modelling by deriving the impedance $Z$ of the panel frame in Eq. (9). Since the wavelength of the flexural wave motion of the panel frame considered here is considerably larger than the LR spacing, the dynamic response of the LRs can be equalized as a frequency dependent effective dynamic mass distributed over the upper face of the MMPP. In accordance with the effective medium method ${ }^{[34]}$, the effective dynamic mass of the $j$ th LR is

$$
m_{\mathrm{eff}, j}(\omega)=\frac{m_{\mathrm{r}, j}}{1-\omega^{2} /\left[\omega_{\mathrm{r}, j}^{2}\left(1+\mathrm{i} \eta_{\mathrm{r}, j}\right)\right]},
$$

in which $\eta_{\mathrm{r}, j}, m_{\mathrm{r}, j}$ and $\omega_{\mathrm{r}, j}$ are the damping loss factor, the static mass and the resonance frequency of the $j$ th $L R$, respectively. The effective dynamic mass density of the MMPP, accounting for $N$ attached LRs, is

$$
\begin{aligned}
& \rho_{\mathrm{eff}}(\omega)=\left(\rho_{\mathrm{p}} S h+\sum_{j=1}^{N} m_{\mathrm{eff}, j}(\omega)\right) \frac{1}{S h} \\
& =\rho_{\mathrm{p}}+\sum_{j=1}^{N} \frac{\rho_{\mathrm{r}, j}}{1-\omega^{2} /\left[\omega_{\mathrm{r}, j}^{2}\left(1+\mathrm{i} \eta_{\mathrm{r}, j}\right)\right]}
\end{aligned}
$$

where $S$ is the surface area of the upper/lower face of the MMPP and $\rho_{\mathrm{p}}$ is the static density of the host panel material. As $\phi<<1$, the influence of the perforations to $\rho_{\mathrm{p}}$ is neglected. An added mass ratio $\xi$ is introduced to define the added mass of the LRs to the host panel, as: 


$$
\xi=\left(\sum_{j=1}^{N} m_{\mathrm{r}, j}\right) / \rho_{\mathrm{p}} S h
$$

The vibroacoustic coupling between the sound pressure excitation and the MMPP as well as the closed cavity is governed by the following dynamic equilibrium equation:

$$
D_{\mathrm{p}} \nabla^{4} w(x, y, t)+\rho_{\mathrm{eff}}(\omega) h \frac{\partial^{2} w(x, y, t)}{\partial t^{2}}=\left(p-p_{D}\right) e^{\mathrm{i} \omega t}
$$

in which $\nabla^{4}=\left(\partial^{2} / \partial x^{2}+\partial^{2} / \partial y^{2}\right)^{2}, \quad D_{\mathrm{p}}=E_{\mathrm{p}} h^{3} / 12\left(1-v_{\mathrm{p}}^{2}\right)$ is the flexural rigidity of the host panel with Young's modulus $E_{\mathrm{p}}$ and Poisson's ratio $v_{\mathrm{p}}$, and $w(x, y, t)$ is the out-of-plane displacement of the host panel. Again, as $\phi<<1$, the effect of perforations on $D_{\mathrm{p}}$ is disregarded. Since a harmonic pressure load is assumed, the displacement field can be expressed as ${ }^{[34]}$ :

$$
w(x, y, t)=w(x, y) e^{\mathrm{i} \omega t}=\sum_{m=1}^{M} \sum_{n=1}^{N} A_{m n} X_{m}(x) Y_{n}(y) e^{\mathrm{i} \omega t},
$$

where $A_{m n}$ and $X_{m}(x) Y_{n}(y)$ are the modal amplitudes and the mode shapes of the $(m, n)$ mode. The velocity can be written as:

$$
v(x, y, t)=\frac{\partial w(x, y, t)}{\partial t}=\sum_{m=1}^{M} \sum_{n=1}^{N} B_{m n} X_{m}(x) Y_{n}(y) e^{\mathrm{i} \omega t},
$$

in which $B_{m n}=\mathrm{i} \omega A_{m n}$. For simply supported boundary conditions, the mode shapes are given by:

$$
\begin{aligned}
& X_{m}(x)=\sin \left(\frac{m \pi x}{a}\right) \\
& Y_{n}(y)=\sin \left(\frac{n \pi y}{b}\right)
\end{aligned}
$$

Substituting Eq. (15) into Eq. (14), replacing $p-p_{\mathrm{D}}$ by $\Delta \bar{p}$ in Eq. (14), and eliminating $e^{\mathrm{i} \omega t}$ gives:

$$
\sum_{m=1}^{M} \sum_{n=1}^{N} Z_{m n} B_{m n} X_{m}(x) Y_{n}(y)=\Delta \bar{p}
$$

in which $Z_{m n}$ is the modal impedance ${ }^{[29]}$ of the $(m, n)^{\text {th }}$ mode, as:

$$
Z_{m n}=\rho_{\text {eff }}(\omega) h \frac{\mathrm{i}\left(\omega^{2}-\omega_{m n}^{2}\right)}{\omega} .
$$


Structural damping is considered in the host panel, resulting in:

$$
Z_{m n}=\rho_{\text {eff }}(\omega) h \frac{\eta_{\mathrm{p}} \omega_{m n} \omega+\mathrm{i}\left(\omega^{2}-\omega_{m n}^{2}\right)}{\omega},
$$

with $\eta_{\mathrm{p}}$ the damping loss factor of the host panel and $\omega_{m n}$ the resonance frequency of the $(m, n)$ mode of the MMPP, given by:

$$
\omega_{m n}=\left[\left(\frac{m \pi}{a}\right)^{2}+\left(\frac{n \pi}{b}\right)^{2}\right] \sqrt{\frac{D_{\mathrm{p}}}{\rho_{\mathrm{eff}}(\omega) h}} .
$$

When adding the identical LRs, the original eigen modes of the FMPP are split into pairs, which are the positive solutions to Eq. (21) in case the resonance frequencies of the LRs coincide with an eigen frequency of the FMPP host panel $\left(\omega_{\mathrm{mn}}=\omega\right)$. The first two eigen frequencies of the MMPP having only $(1,1)$ modes can be expressed as:

$$
\omega_{11}^{\mp}=\frac{\left(\rho_{\mathrm{p}}+N \rho_{\mathrm{r}}+F\right) \mp \sqrt{\left(\rho_{\mathrm{p}}+N \rho_{\mathrm{r}}+F\right)^{2}-4 F \rho_{\mathrm{p}}}}{2 \rho_{\mathrm{p}} / \omega_{\mathrm{r}}^{2}},
$$

in which $F=\left[(\pi / a)^{2}+(\pi / b)^{2}\right]^{2} D_{\mathrm{p}} / h \omega_{\mathrm{r}}^{2}$.

Multiplying both sides of Eq. (18) with $X_{m}(x) Y_{n}(y)$ and integrating over the area of the host panel surface, one gets:

$$
B_{m n}=\frac{\Delta \bar{p} \varepsilon_{m n}}{Z_{m n} \mu_{m n}}
$$

in which ${ }^{[29]}$

$$
\begin{aligned}
& \varepsilon_{m n}=\int_{0}^{b} \int_{0}^{a} X_{m}(x) Y_{n}(y) \mathrm{d} x \mathrm{~d} y \\
& \mu_{m n}=\int_{0}^{b} \int_{0}^{a} X_{m}(x)^{2} Y_{n}(y)^{2} \mathrm{~d} x \mathrm{~d} y
\end{aligned}
$$

Subsequently, the averaged velocity of the panel frame is obtained as:

$$
\begin{aligned}
& \bar{v}=\frac{\int_{0}^{b} \int_{0}^{a} \sum_{m=1}^{M} \sum_{n=1}^{N} B_{m n} X_{m}(x) Y_{n}(y) \mathrm{d} x \mathrm{~d} y}{a b} . \\
& =\Delta \bar{p} \sum_{m=1}^{M} \sum_{n=1}^{N} \frac{\varepsilon_{m n}^{2}}{a b \mu_{m n} Z_{m n}}
\end{aligned}
$$

The impedance of the panel frame can thence be calculated, as: 


$$
Z=\frac{\Delta \bar{p}}{\bar{v}}=\left(\sum_{m=1}^{M} \sum_{n=1}^{N} \frac{\varepsilon_{m n}^{2}}{a b \mu_{m n} Z_{m n}}\right)^{-1} .
$$

At this point, the acoustic properties of the MMPP are entirely theoretically determined.

\section{Full multi-physical simulation model for sound absorption}

Using COMSOL version 5.3a, a full multi-physical finite element method (FEM) simulation model is constructed to evaluate the sound absorption of the proposed MMPP, as shown schematically in Fig. 3. Symmetrical modelling can be exploited for the considered configuration since only normal plane wave incidence is imposed, and no uneven modes of the MMPP and FMPP are excited. More specifically, in the frequency range of interest $(100-1000 \mathrm{~Hz})$ only the fundamental mode $(1,1)$ exists for the FMPP, which is split into two $(1,1)$ modes for theMMPP (elaborated in Section 6.1.2). Exploiting symmetry, only one quarter of the full scale MMPP as well as one quarter of the incidence air part and the backing cavity have to be modeled. Two groups of symmetrical boundaries are used, colored in blue in Fig. 3. The acoustic surface impedance $Z_{\mathrm{s}}$ can be extracted via the average acoustic pressure and the average air particle velocity on the upper surface of the host panel. The sound absorption coefficient of the MMPP can eventually be calculated using Eq. (10).

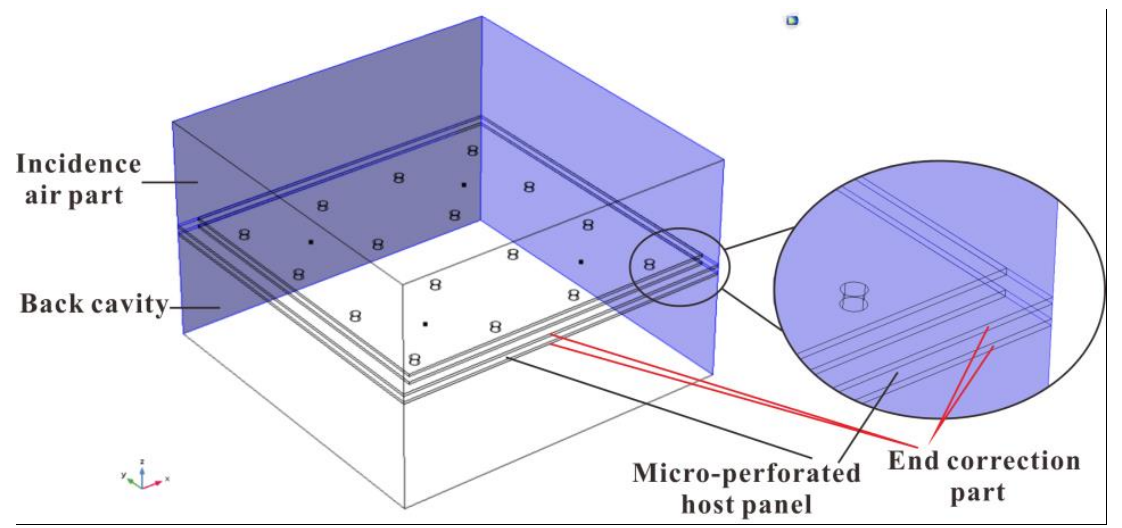

Fig. 3. Schematic of the full simulation model of the MMPP for sound absorption evaluation, in which the components of the end correction part around a symmetrical boundary (colored in blue) are enlarged.

In more detail, the micro-perforated host panel is modeled using the Solid Mechanics module and the incidence air part and the back cavity are modeled using 
the Pressure Acoustics module. The air volume inside the micro-perforations is modeled using the Thermoviscous Acoustics module, which is able to account for the viscous and thermal dissipation inside the viscous and thermal boundary layers adjacent to the perforation walls. Since additional energy dissipation will occur in the domains adjacent to the inlets and outlets of the perforations, two thin layers of Thermoviscous Acoustics are added adjacent to the host panel on either side to allow these dissipation end effects to be taken into account. The radiation-impedance part of the end effects has been considered through the incidence air part and the back cavity. Three kinds of multi-physical interface boundaries are utilized to couple the domains with different properties, including the Thermoviscous Acoustic-Structure Boundary, Acoustic-Structure Boundary and Acoustic-Thermoviscous Acoustic Boundary. On the remaining two boundaries of the incidence air part and the backing cavity a Sound Hard Boundary is imposed.

Since the Thermoviscous Acoustic-Structure Boundary at the interfaces of the host panel and the end correction parts will couple their motions in all three directions, and since the displacement normal to Sound Hard Boundary is fixed as 0, the portions of the end correction parts next to the borders of the host panel are parted, which are highlighted in the enlarged view in Fig. 3, and modeled as Pressure Acoustics in order to set free the displacement constraints of the host panel borders normal to Sound Hard Boundary. Simply supported boundary conditions are realized by fixing the displacements of the upper borders along the $z$-direction. Plane Wave Radiation is used on the upper boundary of the incidence air part to impose the normally impinging plane wave and a specifically defined Impedance boundary is applied to the lower boundary of the backing cavity to account for the remainder of the cavity depth. The LRs are modeled as Point Loads $-Z_{\text {point }} i \omega w$ at the attachment locations of the LRs to the host panel (Fig. 3), with $Z_{\mathrm{point}}=i \omega m_{\mathrm{r}}\left(-i k_{\mathrm{r}} / \omega\right) /\left(i \omega m_{\mathrm{r}}-i k_{\mathrm{r}} / \omega\right)$ and $w$ the displacement of the host panel. By omitting the Point Loads on these points, the proposed simulation model also allows for the computation of the sound absorption of an FMPP. 
To correctly account for the viscous and thermal dissipation, the thickness of the end correction parts equals the thickness of viscous boundary layer at the lowest analyzed frequency $(100 \mathrm{~Hz})$, as the viscous and thermal boundary layer thicknesses decrease with increasing frequency. The smallest mesh size inside the end correction parts and adjacent to the surfaces of the host panel, as well as inside the micro-perforations and adjacent to the perforation walls, should be smaller than the thickness of the viscous boundary layer at the highest frequency $(1000 \mathrm{~Hz})$. The resulting number of degrees of freedom of the simulation model is large $(\sim 3,000,000)$ as viscous and thermal dissipation is considered. The full simulation model computations are performed on the high-performance computing cluster of the Flemish Supercomputer Centre (VSC) in Belgium, using 4 processors (24 cores/processor; Xeon E5-2680v3) and 480 GB of memory.

\section{Finite element unit cell method}

To assess whether the addition of the LRs will introduce a stop band, the wave propagation in the structure needs to be investigated. In literature, wave propagation in metamaterial structures is often investigated by considering the equivalent infinite periodic structure and investigating its dispersion curves based on unit cell modelling ${ }^{[45]}$. The structural unit cell is modeled using FEM, yielding following equations of motion:

$$
\mathbf{D}(\omega) \mathbf{q}=\mathbf{F}
$$

with dynamic stiffness matrix $\mathbf{D}(\omega)$ and generalized displacements $\mathbf{q}$ and forces F . Combining the equations of motion (27) with the Bloch-Floquet periodicity boundary conditions, assuming an infinite two-dimensional (2D) periodic structure, gives rise to an eigenvalue problem in frequency $\omega$ and propagation constants ${ }^{[45]}$ $\mu_{x}$ and $\mu_{y}$

$$
\mathbf{A}\left(\omega, \mu_{x}, \mu_{y}\right) \mathbf{q}^{\text {red }}=\mathbf{0}
$$

in which $\mathbf{q}^{\text {red }}$ is a reduced set of generalized displacements. Solving this eigenvalue problem yields the dispersion curves that describe the wave propagation throughout the infinite periodic structure. 
Stop band behavior is classically assessed using the $\omega(\mu)$ approach by considering an undamped unit cell and imposing a set of real $\left(\mu_{x}, \mu_{y}\right)$, representing freely propagating waves, along the irreducible Brillouin contour (IBC) ${ }^{[45]}$, solving for $\omega$. When damping is present, the $\mu(\omega)$ approach can be used. Complex $\mu$, indicating spatially decaying wave solutions, are then calculated for given real $\omega$ and propagation direction $\theta^{[46,47]}$. In what follows the $\omega(\mu)$ approach is applied to the undamped unit cell to calculate the IBC and assess the stop band behavior. Since damping is present in both the host panel and the LRs of the MMPPs, the influence of damping is analyzed using the $\mu(\omega)$ approach in Section 6.2.2.

In the current study, resonant stop band behavior is obtained by the addition of LRs that can exert a nonzero net force to the host structure on a sub-wavelength scale. Here, the out-of-plane flexural waves are targeted. The considered unit cell is a square $15.875 \mathrm{~mm} \times 15.875 \mathrm{~mm}$ portion of the MMPP, meshed using 2685 quadratic shell elements, with the resonator attached to its center, as shown in Fig. 4 (a). Dispersion curves for the bare FMPP and the MMPP are calculated along the IBC $\left(\mu_{x}, \mu_{y}\right)$ : O $(0,0) \rightarrow \mathrm{A}(\pi, 0) \rightarrow \mathrm{B}(\pi, \pi) \rightarrow \mathrm{O}(0,0)$, as shown schematically in Fig. 4 (b).
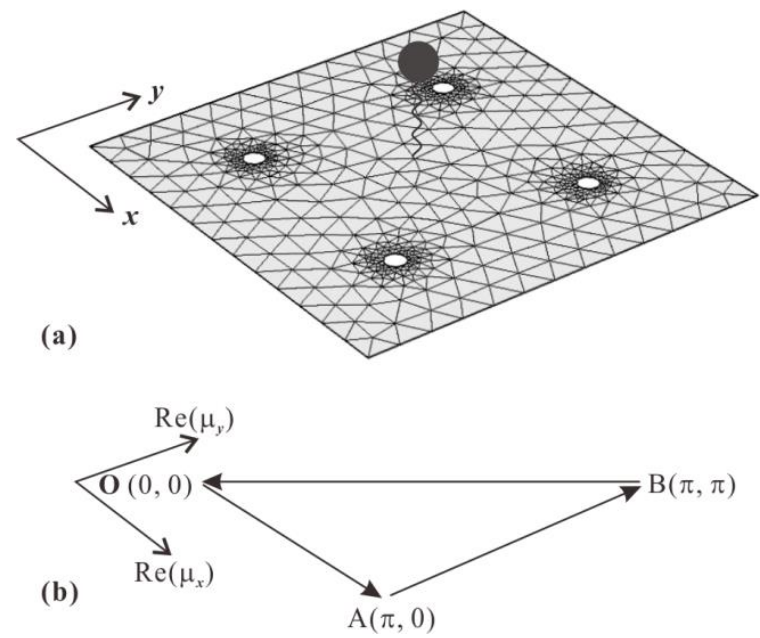

Fig. 4. (a) Unit cell of the MMPP and (b) corresponding IBC in the reciprocal space.

\section{Results and discussions}

\subsection{Model validation and mechanism analysis}

In this section, the developed theoretical model and the full simulation model for sound absorption are validated. The physical mechanism underlying the sound absorption enhancement is analyzed from the perspective of both stop band 
characteristics and acoustic surface impedance.

\subsubsection{Sound absorption enhancement}

In what follows, the results associated with the MMPP are calculated using the parameters listed in Table 1, the FMPP results are obtained with the same MMPP parameters, except for the LRs, and the MPP results are obtained for a rigid MPP with the same perforation diameter and ratio as the MMPP. More specifically, theoretical predictions of the FMPP are calculated using the existing model, proposed by Lee et $a l{ }^{[29]}$. The predicted results for the MPP are based on Maa's model ${ }^{[28]}$, given by Eq. (4) in Section 3.

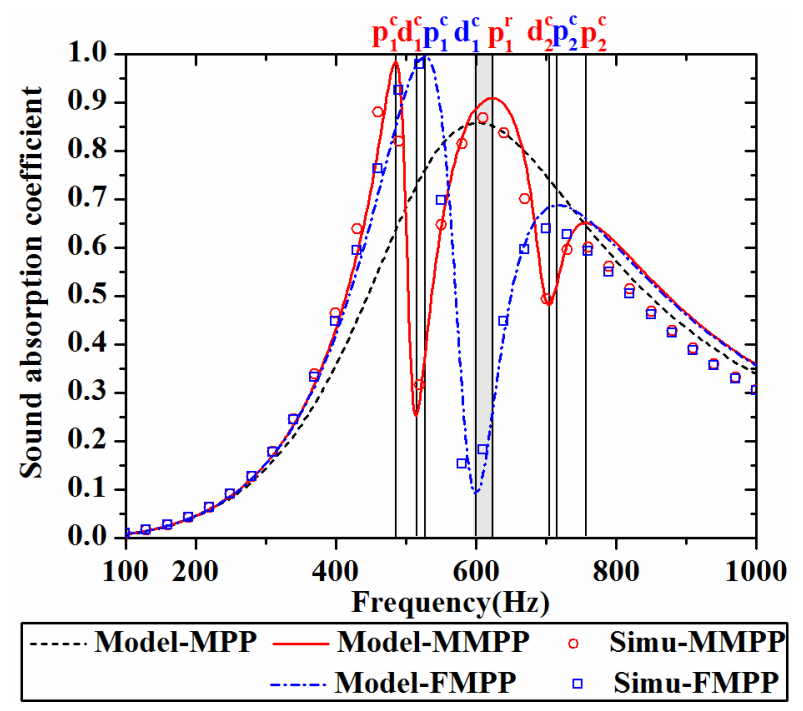

Fig. 5. (Color online) Comparison between model-predicted and simulated sound absorption coefficients for both MMPP and FMPP, with model-predicted results of rigid MPP included as reference. All peaks and dips of MMPP and FMPP are marked as $\mathbf{p}$ or $\mathbf{d}$ along with sequence number subscript and distinguished using superscript of initials of coupling (c) and resonant (r). The stop band of the MMPP is shaded in grey.

The sound absorption coefficients for the MMPP, FMPP and MPP are calculated and illustrated in Fig. 5. The absorption peaks and dips of the MMPP and FMPP are marked with $\mathrm{p}_{1}{ }^{\mathrm{c}}, \mathrm{b}_{1}{ }^{\mathrm{c}}$, etc. in Fig. 5 and in the following Figs. 8 and 9. More specifically, $\mathbf{p}$ stands for peak, $\mathbf{d}$ for dip, superscript $\mathbf{c}$ for coupling, $\mathbf{r}$ for local resonance, the subscript number for peak and dip sequence, and their colors corresponds to the curves' colors. The stop band of the MMPP is shaded in grey, and it will help to explain the sound absorption enhancement in Section 6.1.2. For both the MMPP and FMPP, the model-predicted and simulated sound absorption coefficients agree well and the slight discrepancy between them may come from the averaging operations used in the theoretical model. On the one hand, since the theoretical model 
for the acoustical performance of the FMPP has already been experimentally verified in literature ${ }^{[29]}$, the full simulation model is consequently validated. On the other hand the theoretical model for the MMPP is in its turn validated against the verified full simulation model, considering that the key influence determining the sound absorption performance of the FMPP and MMPP is their vibration behavior, and the LRs in the MMPP are modeled as point forces.

In Fig. 5, the sound absorption coefficient for the MPP is presented as a reference. For the MPP, as its overall acoustic reactance coincides with that of the backing cavity, intensive vibrations of the air particles inside the perforations occur, with the air inside the back cavity functioning as a spring. This results in a distinguished sound absorption peak at $599.18 \mathrm{~Hz}$, named as the pore-cavity peak. When the flexibility of an MPP is taken into account, it changes into an FMPP. Here, both its geometrical and physical parameters are designed in order for its fundamental frequency at $598.91 \mathrm{~Hz}$ to match the pore-cavity peak frequency. Consequently, strong coupling ${ }^{[30]}$ between the pore-cavity resonance and panel-cavity resonance occurs, resulting in high sound absorption peaks, designated as the coupling peaks, before and after the original pore-cavity peak (at $526 \mathrm{~Hz}$ and $716 \mathrm{~Hz}$ ). An undesirable absorption dip, called the coupling dip, also arises at $600 \mathrm{~Hz}$ due to the coupling effect. With the goal of preventing this negative FMPP side effect, the resonance frequency of the MMPP LRs are tuned to be of the mode of the FMPP at $598.91 \mathrm{~Hz}$, with an added mass ratio of $\xi=10 \%$. Consequently, the absorption of the MMPP exhibits an additional enhanced peak, named as the local resonance peak, around the tuned LR resonant frequency.

\subsubsection{Stop band behavior}

Next, the physical mechanism responsible for the sound absorption enhancement of MMPP is analyzed. The dispersion curves for the bare FMPP and the MMPP are presented in Fig. 6. For the MMPP a stop band for the targeted flexural wave mode is obtained between 598.4-627.9 Hz. This stop band is also shaded in grey in Fig. 5, as well as in following Figs. 8 and 9. Therefore, it can be seen from Fig. 5 that the local resonance peak of the MMPP is related to its structural stop band behavior. 


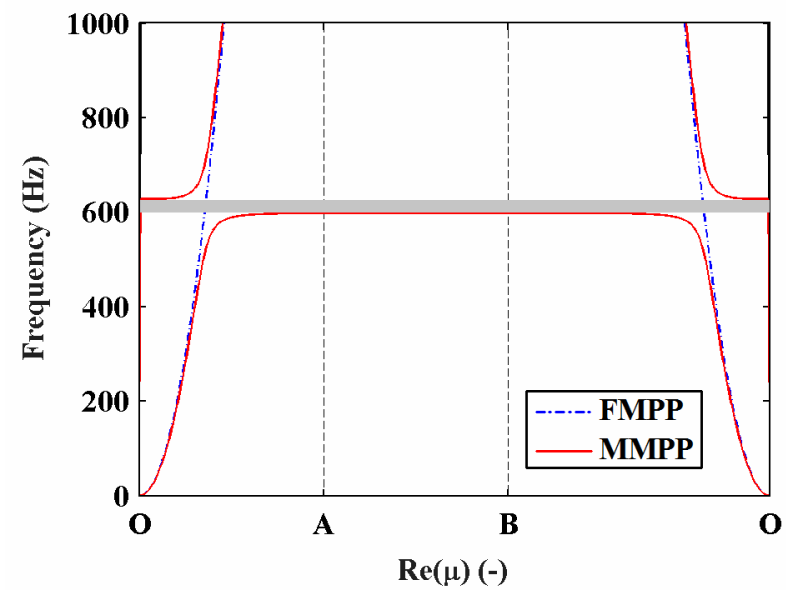

Fig. 6. (Color online) Dispersion curves along the irreducible Brillouin contour for the bare FMPP and the MMPP with $f_{\mathrm{r}}=598.91 \mathrm{~Hz}$ and $\xi=10 \%$, with stop band shaded in grey.

Table 2 Eigen frequencies of the structural modes below $1000 \mathrm{~Hz}$ of the simply supported MMPP sample for $\xi=10 \%$, with $(1,1)$ modes highlighted in bold.

\begin{tabular}{cccccccccc}
\hline \hline Mode & 1 & 2 & 3 & 4 & 5 & 6 & 7 & 8 & 9 \\
\hline$f / \mathrm{Hz}$ & $\mathbf{5 0 9 . 9 7}$ & 593.02 & 593.02 & 596.65 & 597.34 & 597.34 & 597.87 & 597.87 & 597.93 \\
\hline Mode & 10 & 11 & 12 & 13 & 14 & 15 & 16 & 17 & \\
\hline$f / \mathrm{Hz}$ & 597.93 & 598.14 & 598.14 & 598.2 & 598.31 & 598.31 & 598.37 & $\mathbf{6 9 9 . 0 8}$ \\
\hline \hline
\end{tabular}
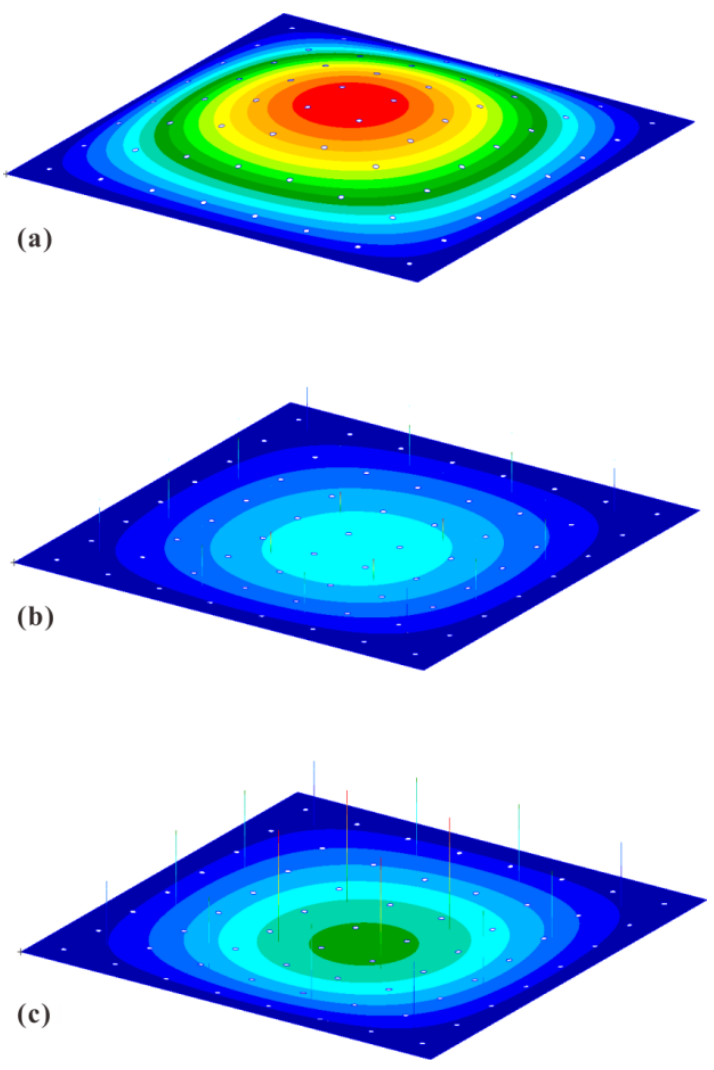

Fig. 7. (Color online) Finite element method calculated (a) first mode of the simply supported bare FMPP sample at $598.91 \mathrm{~Hz}$, (b) first mode of the simply supported MMPP sample at $508.49 \mathrm{~Hz}$ before the stop band and (c) at $696.59 \mathrm{~Hz}$ after the stop band.

The structural eigenmodes of the FMPP and MMPP are analyzed and compared. 
The simply supported bare $63.5 \mathrm{~mm} \times 63.5 \mathrm{~mm}$ FMPP sample has one mode below $1000 \mathrm{~Hz}$, i.e. a $(1,1)$ mode at $598.91 \mathrm{~Hz}$ (see Fig. 7 (a)), with which the coupling dip of the FMPP is associated (see Fig. 5). The mode frequencies for the simply supported $63.5 \mathrm{~mm} \times 63.5 \mathrm{~mm}$ MMPP sample are calculated and given in Table 2. It is confirmed that no modes are present in the predicted stop band frequency range between $598.4-627.9 \mathrm{~Hz}$, as free wave propagation is inhibited in the stop band. This absence of flexural modes prevents the coupling dip in the targeted frequency range. The resonant stop band is known to cause a characteristic mode split, in which the modes before and after the stop band are repeated with in-phase and out-of-phase motion between the LRs and the host structure ${ }^{[36]}$, respectively. As the LRs are tuned to the first mode of the bare sample, 16 modes are retrieved before the stop band for the MMPP sample. The $(1,1)$ mode is found for the MMPP sample at $509.97 \mathrm{~Hz}$ and is repeated with out-of-phase resonator motion after the stop band at $699.08 \mathrm{~Hz}$. These two modes of the MMPP correspond to the two coupling dips of the MMPP shown in Fig. 5. These two fundamental frequencies can also be obtained through Eq. (22), resulting in solutions at $511.65 \mathrm{~Hz}$ and $701.04 \mathrm{~Hz}$, which are in good agreement with the numerical results.

\subsubsection{Acoustic surface resistance and reactance}

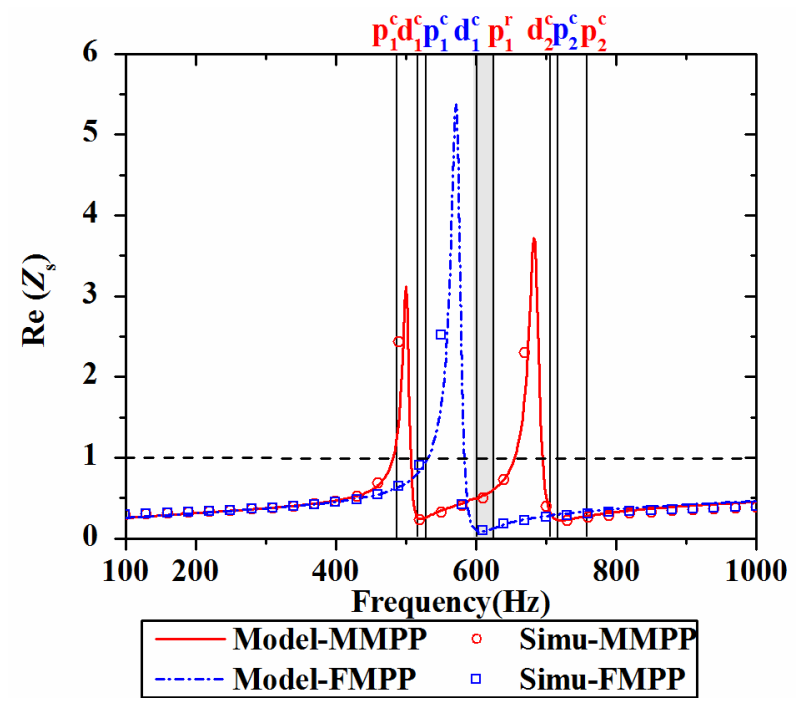

Fig. 8. (Color online) Comparison between model-predicted and simulated normalized acoustic surface resistance for both MMPP and FMPP. 


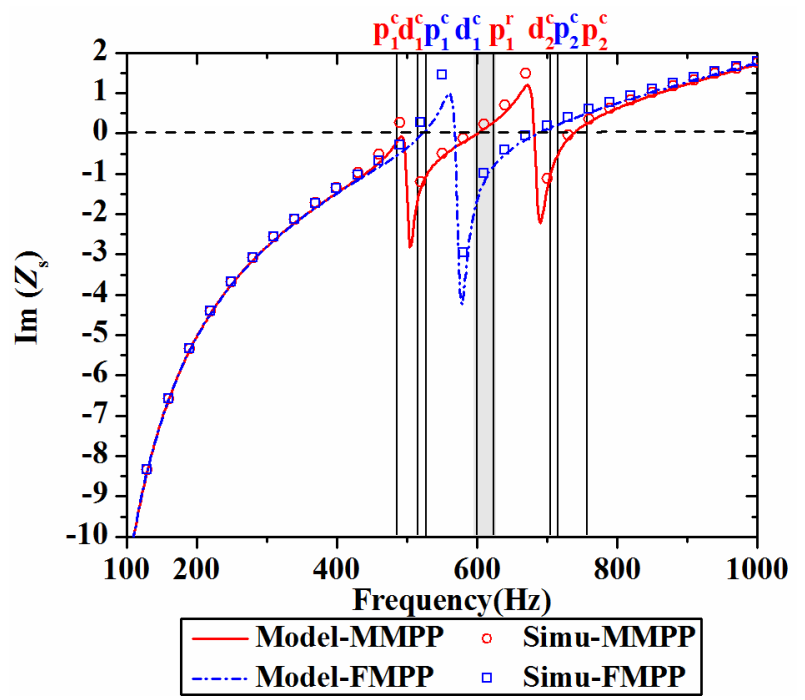

Fig. 9. (Color online) Comparison between model-predicted and simulated normalized acoustic surface reactance for both MMPP and FMPP.

In order to gain deeper insight into the acoustical behavior of the MMPP, the acoustic surface resistance and reactance is analyzed, obtained through Eq. (9) and the proposed full simulation model (Figs. 8 and 9). The good agreement between the model predictions and numerical results again confirm the validity of the theoretical model. From Eq. (10), it can be seen that high sound absorption coefficients require the resistance to approach the characteristic impedance of air $\left(\right.$ i.e. $\left.\operatorname{Re}\left(Z_{\mathrm{s}}\right) \rightarrow 1\right)$ and the reactance to approach 0 (i.e. $\operatorname{Im}\left(Z_{\mathrm{s}}\right) \rightarrow 0$ ), simultaneously. For clarity, the absorption peaks and dips in Fig. 5 are marked accordingly in Figs. 8 and 9. Generally, the two coupling peaks of FMPP (Fig. 5) are in accordance with small absolute value of the reactance (Fig. 9). For the first coupling peak ( $\mathrm{p}_{1}{ }^{\mathrm{c}}$ for MMPP and FMPP), where the sound absorption coefficient approaching 1, the corresponding resistance is close to 1 in Fig. 8. The coupling $\operatorname{dip}\left(\mathrm{d}_{1}{ }^{\mathrm{c}}\right)$ of the FMPP is determined by the extremely small resistance in Fig. 8. Subsequently, the value alterations of corresponding indices (in Figs. 8 and 9) going from FMPP to MMPP explain the accompanying sound absorption improvements. The increased resistance and decreased absolute value of reactance in the stop band frequency range give rise to the local resonance peak $\left(\mathrm{p}_{1}{ }^{\mathrm{r}}\right)$. The two resistance dips of the MMPP (Fig. 8) along with the large absolute reactance value (corresponding to the in-phase and out-of-phase first mode in Fig. 7) after the two reactance dips (Fig. 9), lead to the two coupling dips $\left(\mathrm{d}_{1}{ }^{\mathrm{c}}\right.$ and $\mathrm{d}_{2}{ }^{\mathrm{c}}$ ) of the MMPP ( Fig. 5). The downshift in frequency of the first resistance and reactance peaks of the MMPP compared to the FMPP, results in the corresponding shift of the first coupling peak $\left(\mathrm{p}_{1}{ }^{\mathrm{c}}\right)$ of the MMPP. Moreover, the 
reactance approaching 0 after the additional second reactance dip results in the second coupling peak $\left(\mathrm{p}_{2}{ }^{\mathrm{c}}\right)$ of the MMPP.

\subsection{Influence of key properties of LRs}

While the effects of the host panel properties on the acoustic performance of FMPPs and metamaterial panels have been studied thoroughly ${ }^{[29,30,35,48]}$, the analyses in this section are mainly focused on the influence of the key properties of the LRs on the acoustic performance of the MMPP: the added mass ratio $\xi$, the LR damping factor $\eta_{\mathrm{r}}$ and the use of multiple tuned resonances. Unless otherwise indicated, the geometrical and physical parameters of both the perforated host panel and the LRs are chosen as listed in Table 1.

\subsubsection{Influence of the added mass ratio}

Increasing the added mass of the resonators, while keeping the tuned resonance frequency constant, is known to widen the resonant stop band frequency range ${ }^{[35]}$. This is shown in the dispersion curves of Fig. 10 for the current MMPP unit cell, varying the added mass ratio between $\xi=5 \%, 10 \%$ and $15 \%$, while keeping the resonance frequency at $598.91 \mathrm{~Hz}$. The stop band frequency range decreases to 598.6-613.6 Hz for $\xi=5 \%$, and increases to $598.1-642 \mathrm{~Hz}$ for $\xi=15 \%$.

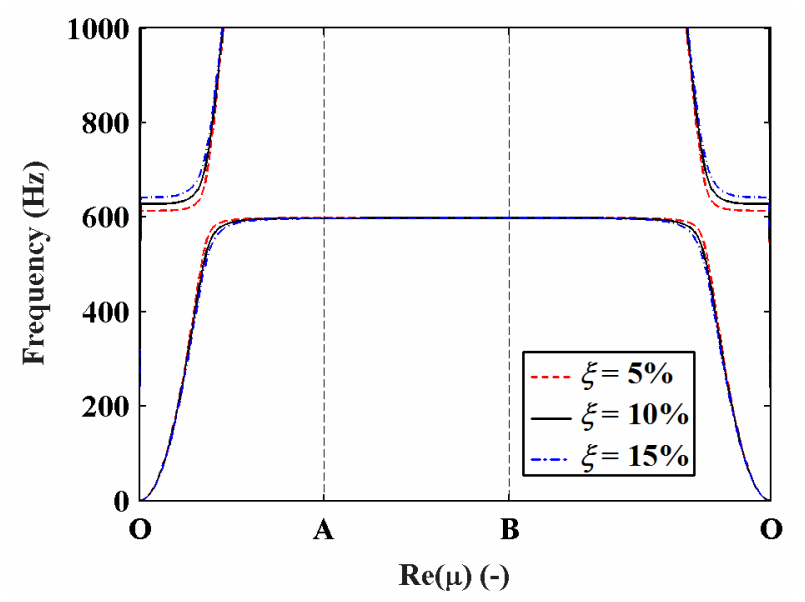

Fig. 10. (Color online) Brillouin contour for the MMPPs with $f_{\mathrm{r}}=598.91 \mathrm{~Hz}$ and added mass ratios $\xi$ $=5 \%, 10 \%$ and $15 \%$.

The structural modes of the simply supported $63.5 \mathrm{~mm} \times 63.5 \mathrm{~mm}$ MMPP sample are reported in Table 3 and 4 for the different mass ratios $\xi=5 \%$ and $15 \%$. These can be compared to the previously calculated structural modes for $\xi=10 \%$ in Table 2. Increasing the mass ratio lowers the eigen frequencies before the stop band and increases the eigen frequencies after the stop band. Inversely, lowering the mass 
ratio increases the eigen frequencies before the stop band and lowers the eigen frequencies after the stop band. The $(1,1)$ mode before and after the stop band occurs at $533.82 \mathrm{~Hz}$ and $667.72 \mathrm{~Hz}$ for $\xi=5 \%$ and at $492.16 \mathrm{~Hz}$ and $723.9 \mathrm{~Hz}$ for $\xi=15 \%$.

Table 3 Eigen frequencies of the structural modes below $1000 \mathrm{~Hz}$ of the simply supported MMPP sample for $\xi=5 \%$, with $(1,1)$ modes highlighted in bold.

\begin{tabular}{cccccccccc}
\hline \hline Mode & 1 & 2 & 3 & 4 & 5 & 6 & 7 & 8 & 9 \\
\hline$f / \mathrm{Hz}$ & $\mathbf{5 3 3 . 8 2}$ & 595.94 & 595.94 & 597.78 & 598.12 & 598.12 & 594.39 & 598.39 & 598.42 \\
\hline Mode & 10 & 11 & 12 & 13 & 14 & 15 & 16 & 17 & \\
\hline$f / \mathrm{Hz}$ & 598.42 & 598.53 & 598.53 & 598.56 & 598.61 & 598.61 & 598.64 & $\mathbf{6 6 7 . 7 2}$ \\
\hline \hline
\end{tabular}

Table 4 Eigen frequencies of the structural modes below $1000 \mathrm{~Hz}$ of the simply supported MMPP sample for $\xi=15 \%$, with $(1,1)$ modes highlighted in bold.

\begin{tabular}{cccccccccc}
\hline \hline Mode & 1 & 2 & 3 & 4 & 5 & 6 & 7 & 8 & 9 \\
\hline$f / \mathrm{Hz}$ & $\mathbf{4 9 2 . 1 6}$ & 590.15 & 590.15 & 595.53 & 596.56 & 596.56 & 597.35 & 597.35 & 597.44 \\
\hline Mode & 10 & 11 & 12 & 13 & 14 & 15 & 16 & 17 & \\
\hline$f / \mathrm{Hz}$ & 597.44 & 597.76 & 597.76 & 597.85 & 598.01 & 598.01 & 598.1 & $\mathbf{7 2 3 . 9}$ & \\
\hline \hline
\end{tabular}

Correspondingly, the influence of the added mass ratio on the sound absorption coefficient curves, as well as the acoustic surface resistance and reactance, is analyzed (Figs. 11, 12 and 13). As illustrated in Fig. 11, the local resonance peak of MMPP is broadened with increasing added mass ratio, due to the widening of the stop band frequency range (Fig. 10). However, the resulting decline in resistance (Fig. 12) causes a slight decrease of the peak value. The downshift in frequency of the first coupling peak and the upshift in frequency of the second coupling peak are related to the corresponding shifts of peaks and dips in the resistance (Fig. 12) and reactance (Fig. 13) curves, in accordance with the shifting $(1,1)$ modes (Tables 2, 3 and 4). As for the peak/dip value alteration, the reduced magnitude of the two coupling peaks results from the enlargement of the absolute reactance values. The decrease of the absorption magnitude at the first coupling dip and the increase of the absorption magnitude at the second coupling dip are caused by the decrease and increase of resistance, respectively. 


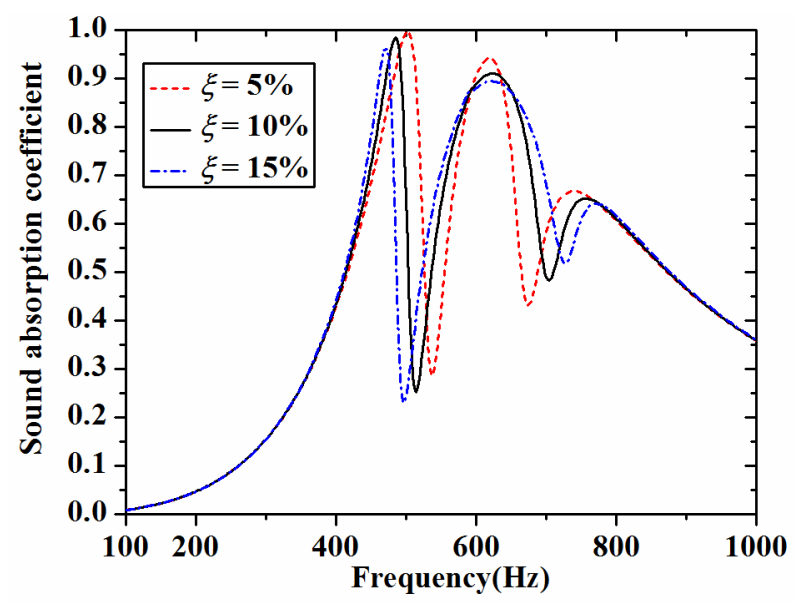

Fig. 11. (Color online) Sound absorption coefficients as a function of frequency for the MMPPs for varying added mass ratios for the LRs.

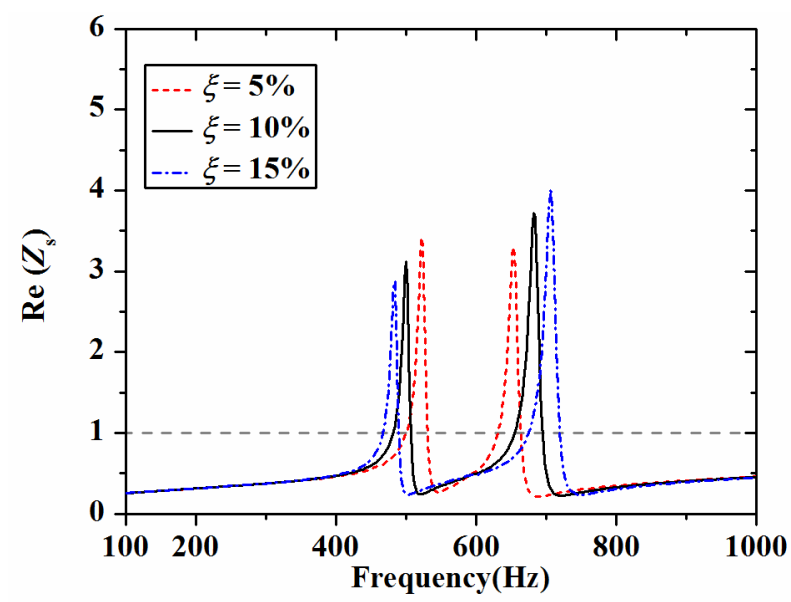

Fig. 12. (Color online) Model predicted acoustic surface resistance as a function of frequency for the MMPPs for varying added mass ratios for the LRs.

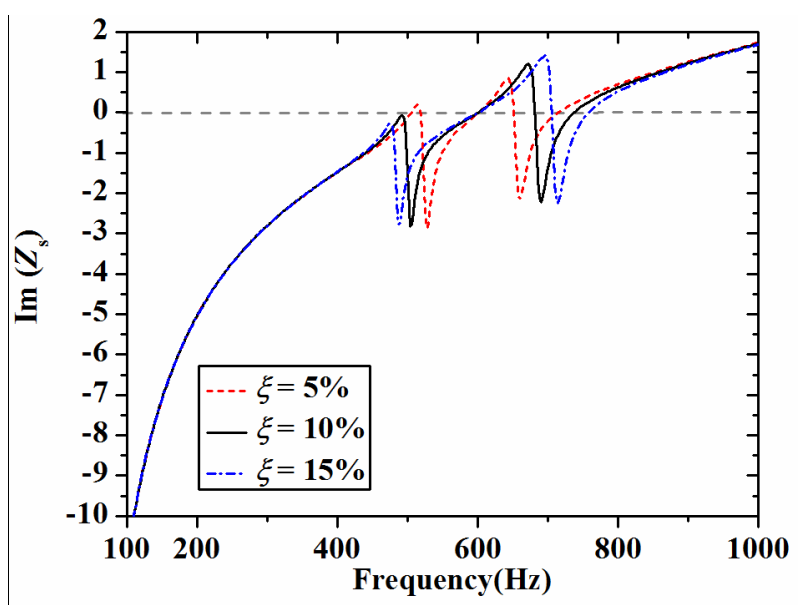

Fig. 13. (Color online) Model predicted acoustic surface reactance as a function of frequency for the MMPPs for varying added mass ratios for the LRs. 


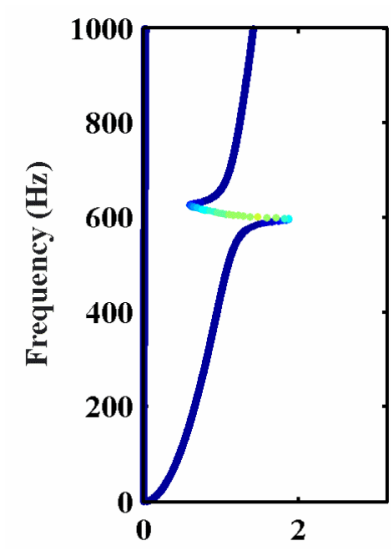

(a)

$\operatorname{Re}(\mu)(-)$

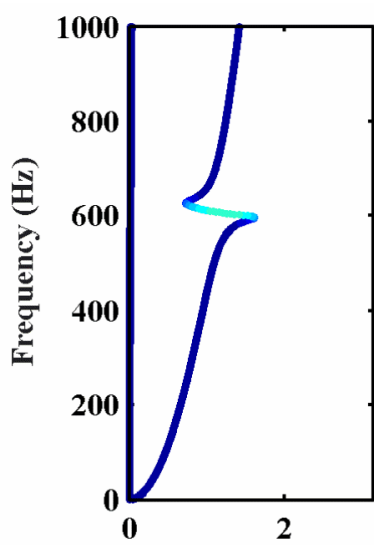

(b)

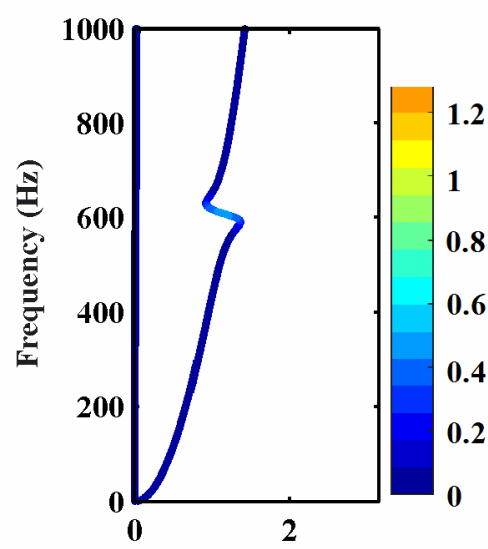

(c)

Fig. 14. (Color online) Dispersion curves along the OA direction for the damped MMPP unit cell with LR damping factors $\eta_{\mathrm{p}}=0.5 \%$ and $\eta_{\mathrm{r}}=$ (a) $1 \%$, (b) $2 \%$, and (c) $5 \%$, colored according to the magnitude of the spatial decay $|\operatorname{Im}(\mu)|$

The addition of damping to the resonant additions of a locally resonant metamaterial is known to broaden the frequency range of attenuation at the expense of peak attenuation performance ${ }^{[35,47,49]}$. Damping is included in the considered MMPP structure by considering a complex spring constant for the resonator $k_{\mathrm{r}}^{\prime}=k_{\mathrm{r}}\left(1+\mathrm{i} \eta_{\mathrm{r}}\right)$, as included in the effective medium method of Eq. (11). The damping in the resonator is varied between $\eta_{\mathrm{r}}=1 \%, 2 \%$ and $5 \%$. A structural damping of $\eta_{\mathrm{p}}=0.5 \%$ is also included in the host structure, which is known to mainly affect the frequency region outside the resonant stop band. Dispersion curves are calculated using the $\mu(\omega)$ approach along the OA propagation direction, corresponding to $\theta=0^{\circ}$, in Fig. 14 . Complex propagation constants $\mu$ are obtained, with $\operatorname{Re}(\mu)$ and $\operatorname{Im}(\mu)$ denoting the propagative and decaying part. As reported in Reference ${ }^{[38,49]}$ for these damped dispersion curves, increasing $\eta_{\mathrm{r}}$ causes the stop band to close gradually. The frequency range of attenuation is slightly widened around the original stop band, which will strongly attenuate the modes in the vicinity of the stop band, at the expense of peak attenuation performance inside the stop band. Increasingly more of the previously freely propagating flexural modes before and after the stop band also vanish with increasing closing of the dispersion curves due to damping in the resonator. 


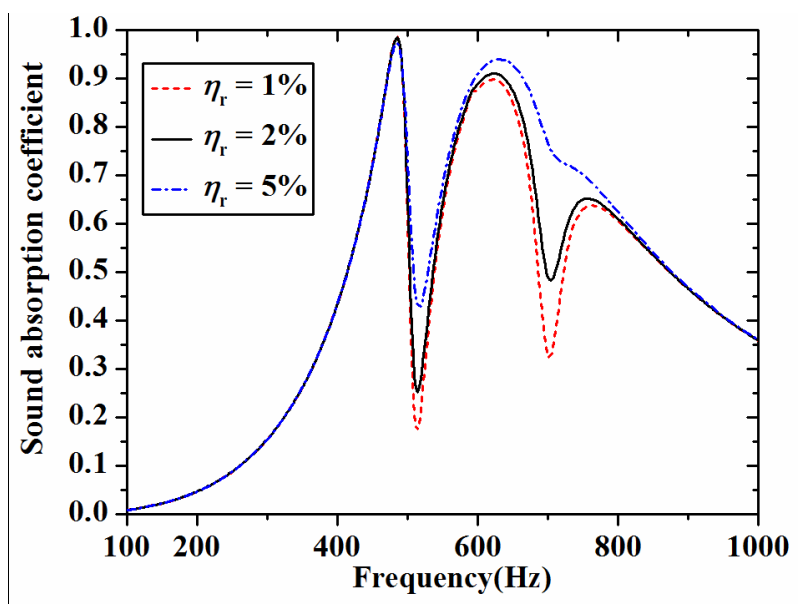

Fig. 15. (Color online) Sound absorption coefficients as a function of frequency for the MMPPs for varying LR damping factors for LRs.

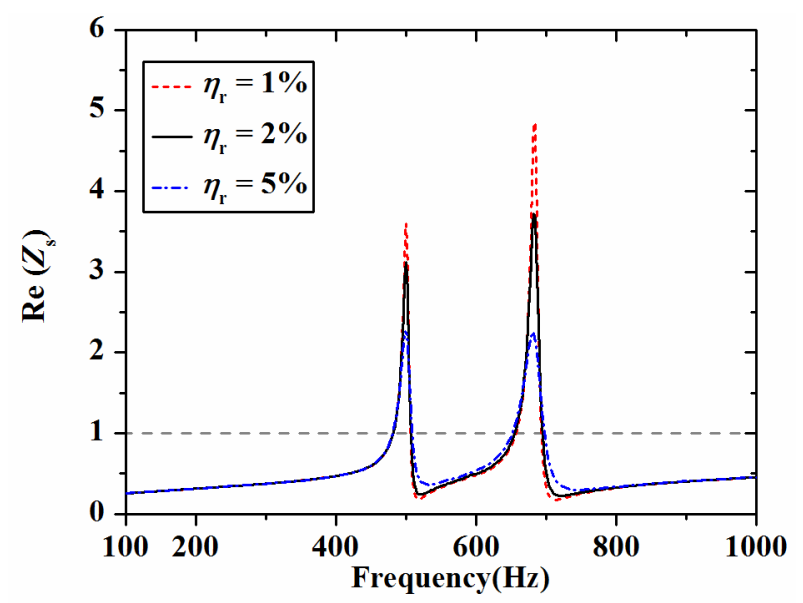

Fig. 16. (Color online) Model predicted acoustic surface resistance as a function of frequency for the MMPPs for varying LR damping factors.

The corresponding influence of the damping of the LRs on the sound absorption enhancement of the MMPP is now examined. The sound absorption coefficient curves are given in Fig. 15, and the associated acoustic surface resistance and reactance are shown in Fig. 16 and 17, respectively. For an increasing damping factor, the absorption at the coupling dips is remarkably improved, due to the increase of both resistance (Fig. 16) and reactance (Fig. 17) resulting from less pronounced panel vibrations at the $(1,1)$ modes. The locations of the coupling dips remain unchanged, however, as increasing damping does not alter the $(1,1)$ mode frequencies. Meanwhile, the local resonance peak is enhanced and widened slightly, resulting from the broadening of the frequency range of attenuation (Fig. 14) and the increase of resistance (Fig. 16). Because of the same effects, the absorption of the second coupling peak is increased notably. Nevertheless, the first coupling peak is barely sensitive to the change in damping, because of the limited influence of the resonator 
damping for frequencies far away from the stop band, as observed in the dispersion curves of Fig. 14. Consequently, the sound absorption enhancement of the MMPP may be further improved with increasing damping of the LRs (Fig. 15).

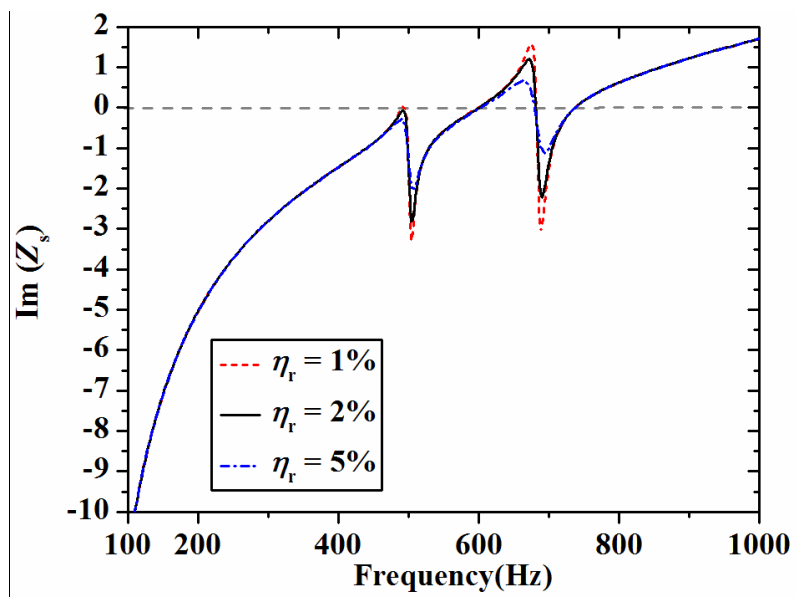

Fig. 17. (Color online) Model predicted acoustic surface reactance as a function of frequency for the MMPPs for varying LR damping factors.

\subsubsection{Influence of the multiple resonances}

In this section, LRs with multiple resonances are applied to examine the further improvement potential of local resonances on the sound absorption. One more group of 16 LRs tuned to $508.49 \mathrm{~Hz}$ (corresponding to the first coupling dip for the MMPP in Fig. 5) is added at the same locations of the original LRs tuned to $598.91 \mathrm{~Hz}$ to realize double resonances. Another additional group of $16 \mathrm{LRs}$ tuned to $696.59 \mathrm{~Hz}$ (corresponding to the second coupling dip for MMPP in Fig. 5) is added at the same locations to achieve triple resonances. The total added mass ratio for the double and triple resonances is fixed to $10 \%$, resulting in $1 / 2$ or $1 / 3$ mass per resonator as compared to the single resonance LR, for the double and triple resonance cases respectively. In reality, one of the possible ways to realize multiple resonances through this kind of parallel connection has been validated in Reference [38]. Due to the mass-cantilever resonant structures' actual shape (especially the cross-sectional shape of the cantilever) and physiccal properties (i.e. density, modulus and Poisson's ratio), they may have more than one mode that can cause stop band behaviors. The dispersion curves of the MMPPs with LRs of single, double and triple resonance are shown in Fig. 18. Two stop bands (508.32-518.93 Hz and 598.64-615.34 Hz) emerge for double resonance case, and three stop bands $(508.38-515.46 \mathrm{~Hz}, 598.73-608.17$ $\mathrm{Hz}$ and $696.30-709.50 \mathrm{~Hz}$ ) for the triple resonance case, respectively. For both cases, the multiple stop bands are separated by pass bands, in which structural modes are 
again allowed.

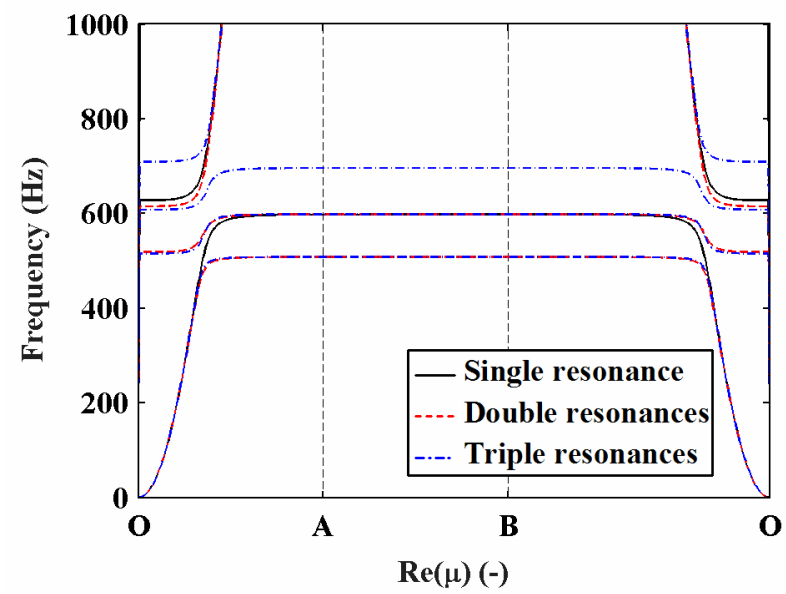

Fig. 18. (Color online) Brillouin contour for the MMPPs with LRs of single, double and triple resonance.

The structural modes of the MMPP samples with LRs of double and triple resonance are given in Table 5 and 6. Compared to the previously calculated structural modes for the single resonance case (Table 2), additional $(1,1)$ modes occur in the pass bands in between the stop bands created by the multiple resonances. For the double-resonance case, the $(1,1)$ modes occur at $477.15 \mathrm{~Hz}, 558.21 \mathrm{~Hz}$ and 680.4 Hz. For the triple-resonance case, the $(1,1)$ modes occur at $483.35 \mathrm{~Hz}, 554.81 \mathrm{~Hz}$, $635.68 \mathrm{~Hz}$ and $739.77 \mathrm{~Hz}$. These $(1,1)$ modes, except for the mode at $739.77 \mathrm{~Hz}$ for the triple resonance case, correspond to sound absorption dips (Fig. 19). The reason for this exception might be the large difference of $739.77 \mathrm{~Hz}$ from the pore-cavity resonance frequency $599.18 \mathrm{~Hz}$ for the rigid MPP. In literature ${ }^{[29]}$, the $(1,1)$ mode of FMPP occuring after the pore-cavity resonance peak always leads to additional absorption peaks. From this observation, a broad stop band, leading to $(1,1)$ modes on either side of the original MPP panel absorption peak, could lead to even broader absorption frequency ranges.

Table 5 Eigen frequencies of the structural modes below $1000 \mathrm{~Hz}$ of the simply supported MMPP sample with LRs of double resonance, with $(1,1)$ modes highlighted in bold.

\begin{tabular}{cccccccccc}
\hline \hline Mode & 1 & 2 & 3 & 4 & 5 & 6 & 7 & 8 & 9 \\
\hline$f / \mathrm{Hz}$ & $\mathbf{4 7 7 . 1 5}$ & 506.71 & 506.71 & 507.8 & 508.01 & 508.01 & 508.17 & 508.17 & 508.19 \\
\hline Mode & 10 & 11 & 12 & 13 & 14 & 15 & 16 & 17 & 18 \\
\hline$f / \mathrm{Hz}$ & 508.19 & 508.25 & 508.25 & 508.27 & 508.31 & 508.31 & 508.32 & $\mathbf{5 5 8 . 2 1}$ & 596.01 \\
\hline Mode & 19 & 20 & 21 & 22 & 23 & 24 & 25 & 26 & 27 \\
\hline$f / \mathrm{Hz}$ & 596.01 & 597.79 & 598.13 & 598.13 & 598.39 & 598.39 & 598.42 & 598.42 & 598.53 \\
\hline Mode & 28 & 29 & 30 & 31 & 32 & 33 & & & \\
\hline$f / \mathrm{Hz}$ & 598.53 & 598.56 & 598.61 & 598.61 & 598.64 & $\mathbf{6 8 0 . 4}$ & & \\
\hline \hline
\end{tabular}


Table 6 Eigen frequencies of the structural modes below $1000 \mathrm{~Hz}$ of the simply supported MMPP sample with LRs of triple resonance, with $(1,1)$ modes highlighted in bold.

\begin{tabular}{cccccccccc}
\hline \hline Mode & 1 & 2 & 3 & 4 & 5 & 6 & 7 & 8 & 9 \\
\hline$f$ & $\mathbf{4 8 3 . 3 5}$ & 507.3 & 507.3 & 508.03 & 508.17 & 508.17 & 508.28 & 508.28 & 508.29 \\
\hline Mode & 10 & 11 & 12 & 13 & 14 & 15 & 16 & 17 & 18 \\
\hline$f$ & 508.29 & 508.33 & 508.33 & 508.35 & 508.37 & 508.37 & 508.38 & $\mathbf{5 5 4 . 8 1}$ & 596.61 \\
\hline Mode & 19 & 20 & 21 & 22 & 23 & 24 & 25 & 26 & 27 \\
\hline$f$ & 596.91 & 598.15 & 598.39 & 598.39 & 598.56 & 598.56 & 598.58 & 598.58 & 598.65 \\
\hline Mode & 28 & 29 & 30 & 31 & 32 & 33 & 34 & 35 & 36 \\
\hline$f$ & 598.65 & 598.68 & 598.71 & 598.71 & 598.73 & $\mathbf{6 3 5 . 6 8}$ & 693.38 & 693.38 & 659.39 \\
\hline Mode & 37 & 38 & 39 & 40 & 41 & 42 & 43 & 44 & 45 \\
\hline$f$ & 695.76 & 695.76 & 696.04 & 696.04 & 696.08 & 696.08 & 696.19 & 696.19 & 696.22 \\
\hline Mode & 46 & 47 & 48 & 49 & & & & & \\
\hline$f$ & 696.28 & 696.28 & 696.31 & $\mathbf{7 3 9 . 7 7}$ & & & & & \\
\hline \hline
\end{tabular}

The sound absorption coefficient, acoustic surface resistance and reactance are illustrated as Fig. 19, 20 and 21. Absorption coupling dips are converted to resonance peaks in the corresponding stop band regions caused by the additionally added LRs. Meanwhile, two new coupling dips appear before and after the original ones, respectively, which is also caused by a mode split as for the single resonance LR (Fig. 7). Correspondingly, extra peaks and dips occur in the acoustic surface resistance (Fig. 20) and reactance (Fig. 21). Therefore, additional combinations of sufficient resistance (i.e. $\operatorname{Re}\left(Z_{\mathrm{s}}\right) \rightarrow 1$ ) and appropriate reactance (i.e. $\operatorname{Im}\left(Z_{\mathrm{s}}\right) \rightarrow 0$ ) are realized and the sound absorption of the MMPP is further improved. Multiple sound absorption peaks appear, with peak value larger than 0.9 and in a relatively broad frequency range, while the smallest total thickness-to-wavelength ratio is $\sim 1 / 15$. Comparatively, the smallest thickness-to-wavelength ratio for the original MPP and FMPP in Fig. 5 is $\sim 1 / 12$ and $\sim 1 / 13$, respectively. It is noted that the LRs in this paper are added on the cavity side (Fig. 2), which helps to keep the whole structure compact. 


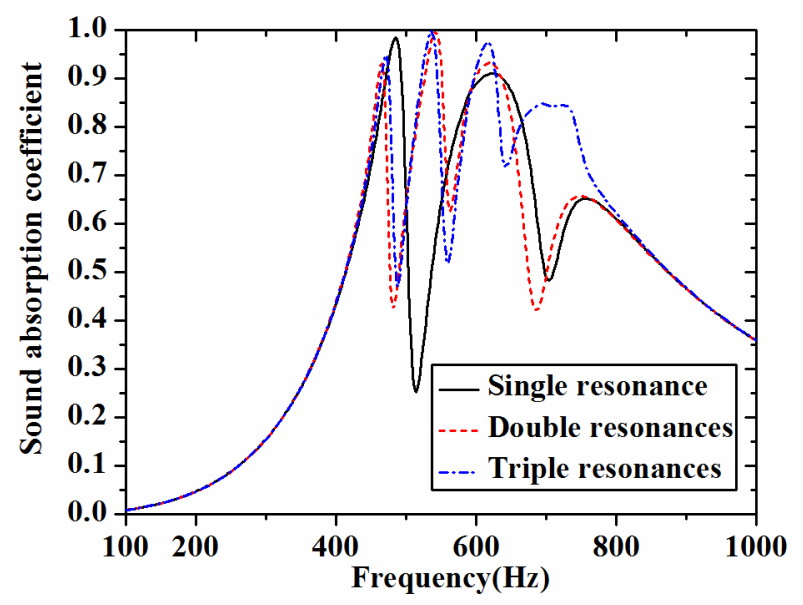

Fig. 19. (Color online) Sound absorption coefficients as a function of frequency for MMPPs with LRs of single, double and triple resonance.

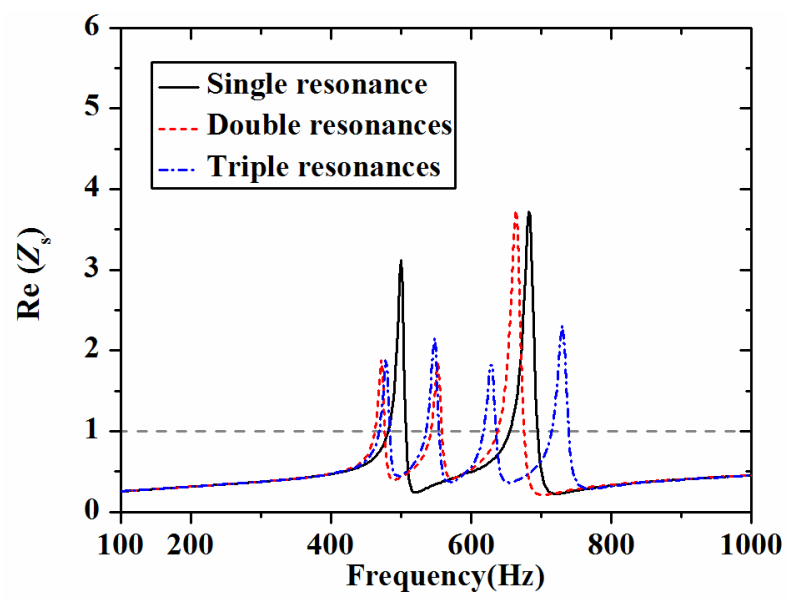

Fig. 20. (Color online) Model predicted acoustic surface resistance as a function of frequency for MMPPs with LRs of single, double and triple resonance.

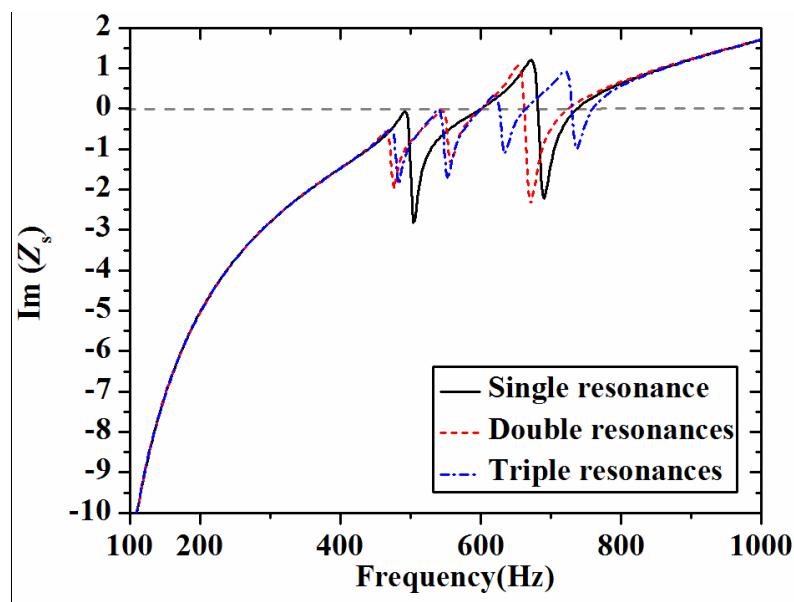

Fig. 21. (Color online) Model predicted acoustic surface reactance as a function of frequency for MMPPs with LRs of single, double and triple resonance. 


\section{Conclusions}

In order to enhance the sound absorption performance of flexible micro-perforated panels (FMPPs), suffering from panel-cavity coupling absorption dips, a metamaterial-based micro-perforated panel (MMPP) is proposed by attaching local resonators (LRs) on a sub-wavelength scale to an FMPP, targeting the structural out-of-plane flexural waves. A theoretical model based on the equivalent medium method and a full simulation model using multi-physical coupling are developed to study the sound absorption performance of this new structure. A finite element unit cell method is utilized to evaluate the structural stop band behavior of the MMPP. An outspoken sound absorption enhancement is demonstrated both theoretically and numerically. The underlying mechanism is the acoustic surface impedance improvement resulting from the flexural wave stop band behavior. By using the proposed theoretical model, the influence of the key properties of the LRs (i.e. added mass ratio, damping and multiple resonances) on the MMPP sound absorption enhancement is analyzed, accompanied with the analysis of the stop band behavior and structural modes. It is found that increasing the added mass ratio and damping of the LRs, as well as adopting multiple local resonances, can further increase and broaden the absorption enhancement. Eventually, a practical balance between broad working ranges and thin material/structure thickness is achieved. The proposed MMPP shows great potential in noise control industry.

\section{Acknowledgements}

This work is supported by the Fundamental Research Funds for the Central Universities (0300-G2018KY0307), the National Natural Science Foundation of China (11772248, U1737107 and 11761131003), DFG (ZH15/32-1) and the Shaanxi Foundation for Selected Overseas Chinese (2017025). Ren is supported by China Scholarship Council as a visiting PhD student to KU Leuven. The research of L. Van Belle is funded by a doctoral grant from the Research Foundation - Flanders $(11 \mathrm{ZH} 817 \mathrm{~N})$. E. Deckers is a postdoctoral fellow of the Research Foundation Flanders (12D2614N). The authors gratefully acknowledge the KU Leuven Research Fund for their support.

\section{References}


[1] Craster RV, Guenneau S. Acoustic Metamaterials: Negative Refraction, Imaging, Lensing and Cloaking: Springer Science \& Business Media, 2012.

[2] Nobrega ED, Gautier F, Pelat A, et al. Vibration band gaps for elastic metamaterial rods using wave finite element method. Mechanical Systems and Signal Processing, 2016, 79: 192-202.

[3] Abdeljaber O, Avci O, Kiranyaz S, et al. Optimization of linear zigzag insert metastructures for low-frequency vibration attenuation using genetic algorithms. Mechanical Systems and Signal Processing, 2017, 84: 625-641.

[4] Li J, Chan C. Double-negative acoustic metamaterial. Physical Review E, 2004, $70(5)$.

[5] Lee SH, Park CM, Seo YM, et al. Acoustic metamaterial with negative density. Physics Letters A, 2009, 373 (48): 4464-4469.

[6] Fang N, Xi D, Xu J, et al. Ultrasonic metamaterials with negative modulus. Nat Mater, 2006, 5 (6): 452-456.

[7] Hladky-Hennion AC, Vasseur JO, Haw G, et al. Negative refraction of acoustic waves using a foam-like metallic structure. Applied Physics Letters, 2013, 102 (14): 144103.

[8] Popa B-I, Cummer SA. Homogeneous and compact acoustic ground cloaks. Physical Review B, 2011, 83 (22).

[9] Chen H, Chan CT. Acoustic cloaking in three dimensions using acoustic metamaterials. Applied Physics Letters, 2007, 91 (18): 183518.

[10]Hawkes AM, Katko AR, Cummer SA. A microwave metamaterial with integrated power harvesting functionality. Applied Physics Letters, 2013, 103 (16): 163901.

[11]Zhu J, Christensen J, Jung J, et al. A holey-structured metamaterial for acoustic deep-subwavelength imaging. Nature Physics, 2011, 7 (1): 52-55.

[12]Ward GP, Lovelock RK, Murray AR, et al. Boundary-Layer Effects on Acoustic Transmission Through Narrow Slit Cavities. Phys Rev Lett, 2015, 115 (4): 044302.

[13]Molerón M, Serra-Garcia M, Daraio C. Visco-thermal effects in acoustic metamaterials: from total transmission to total reflection and high absorption. New Journal of Physics, 2016, 18 (3): 033003.

[14]Ruiz H, Claeys C, Deckers E, et al. Numerical and experimental study of the effect of microslits on the normal absorption of structural metamaterials. Mechanical Systems and Signal Processing, 2016, 70-71: 904-918.

[15]Liu Z-M, Pang Y. Effect of the size and pressure on the modified viscosity of water in microchannels. Acta Mechanica Sinica, 2015, 31 (1): 45-52.

[16]Azma S, Rezazadeh G, Shabani R, et al. Viscous fluid damping in a laterally oscillating finger of a comb-drive micro-resonator based on micro-polar fluid theory. Acta Mechanica Sinica, 2016, 32 (3): 397-405.

[17]Liang Z, Li J. Extreme Acoustic Metamaterial by Coiling Up Space. Physical Review Letters, 2012, 108 (11).

[18]Cai X, Guo Q, Hu G, et al. Ultrathin low-frequency sound absorbing panels based on coplanar spiral tubes or coplanar Helmholtz resonators. Applied Physics Letters, 2014, 105 (12): 121901. 
[19]Li Y, Assouar BM. Acoustic metasurface-based perfect absorber with deep subwavelength thickness. Applied Physics Letters, 2016, 108 (6): 063502.

[20]Christensen J, Romero-Garcia V, Pico R, et al. Extraordinary absorption of sound in porous lamella-crystals. Sci Rep, 2014, 4: 4674.

[21]Ren SW, Meng H, Xin FX, et al. Ultrathin multi-slit metamaterial as excellent sound absorber: Influence of micro-structure. Journal of Applied Physics, 2016, 119 (1): 014901.

[22]Li R-Q, Zhu X-F, Liang B, et al. A broadband acoustic omnidirectional absorber comprising positive-index materials. Applied Physics Letters, 2011, 99 (19): 193507.

[23]Climente A, Torrent D, Sánchez-Dehesa J. Omnidirectional broadband acoustic absorber based on metamaterials. Applied Physics Letters, 2012, 100 (14): 144103.

[24] Wei P, Croënne C, Tak Chu S, et al. Symmetrical and anti-symmetrical coherent perfect absorption for acoustic waves. Applied Physics Letters, 2014, 104 (12): 121902.

[25] Song JZ, Bai P, Hang ZH, et al. Acoustic coherent perfect absorbers. New Journal of Physics, 2014, 16 (3): 033026.

[26] Song GY, Cheng Q, Huang B, et al. Broadband fractal acoustic metamaterials for low-frequency sound attenuation. Applied Physics Letters, 2016, 109 (13): 131901.

[27]Romero-Garcia V, Theocharis G, Richoux O, et al. Perfect and broadband acoustic absorption by critically coupled sub-wavelength resonators. Sci Rep, 2016, 6: 19519.

[28] Maa D-Y. Potential of microperforated panel absorber. J Acoust Soc Am, 1998, 104 (5): 2861-2866.

[29]Lee YY, Lee EWM, Ng CF. Sound absorption of a finite flexible micro-perforated panel backed by an air cavity. Journal of Sound and Vibration, 2005, 287 (1-2): 227-243.

[30]Bravo T, Maury C, Pinhede C. Vibroacoustic properties of thin micro-perforated panel absorbers. J Acoust Soc Am, 2012, 132 (2): 789-798.

[31]Wang C, Cheng L, Pan J, et al. Sound absorption of a micro-perforated panel backed by an irregular-shaped cavity. J Acoust Soc Am, 2010, 127 (1): 238-246.

[32] Wang C, Huang L. On the acoustic properties of parallel arrangement of multiple micro-perforated panel absorbers with different cavity depths. J Acoust Soc Am, 2011, 130 (1): 208-218.

[33]Bravo T, Maury C, Pinhede C. Enhancing sound absorption and transmission through flexible multi-layer micro-perforated structures. J Acoust Soc Am, 2013, 134 (5): 3663-3673.

[34]Xiao Y, Wen J, Wen X. Sound transmission loss of metamaterial-based thin plates with multiple subwavelength arrays of attached resonators. Journal of Sound and Vibration, 2012, 331 (25): 5408-5423.

[35]Claeys C, Vergote K, Sas P, et al. On the potential of tuned resonators to obtain low-frequency vibrational stop bands in periodic panels. Journal of Sound and 
Vibration, 2013, 332 (6): 1418-1436.

[36]Claeys C, Sas P, Desmet W. On the acoustic radiation efficiency of local resonance based stop band materials. Journal of Sound and Vibration, 2014, 333 (14): 3203-3213.

[37]Claeys C, Deckers E, Pluymers B, et al. A lightweight vibro-acoustic metamaterial demonstrator: Numerical and experimental investigation. Mechanical Systems and Signal Processing, 2016, 70-71: 853-880.

[38] Van Belle L, Claeys C, Deckers E, et al. On the impact of damping on the dispersion curves of a locally resonant metamaterial: Modelling and experimental validation. Journal of Sound and Vibration, 2017, 409: 1-23.

[39]ISO10534-1998. Acousitcs determination of sound absorption coefficient and impedance in impedance tubes - Part 2: Transfer-function method. 1998.

[40]Morse PM, Ingard KU. Theoretical acoustics: Princeton university press, 1968.

[41]Xin FX, Lu TJ. Generalized Method to Analyze Acoustomechanical Stability of Soft Materials. Journal of Applied Mechanics, 2016, 83 (7): 071004.

[42]Xin FX, Lu TJ. Acoustomechanical constitutive theory for soft materials. Acta Mechanica Sinica, 2016, 32 (5): 828-840.

[43]Xin FX, Lu TJ. Acoustomechanical giant deformation of soft elastomers with interpenetrating networks. Smart Materials and Structures, 2016, 25 (7): 07LT02.

[44]Xin FX, Lu TJ. A nonlinear acoustomechanical field theory of polymeric gels. International Journal of Solids and Structures, 2017, 112: 133-142.

[45]Brillouin L. Wave propagation in periodic structures: McGraw-Hill Book Company, 1946.

[46]Mace BR, Manconi E. Modelling wave propagation in two-dimensional structures using finite element analysis. Journal of Sound and Vibration, 2008, 318 (4-5): 884-902.

[47]Van Belle L, Claeys C, Deckers E, et al. On the impact of damping on the dispersion curves of a locally resonant metamaterial: modelling and experimental validation. submitted to Journal of Sound and Vibration, 2017.

[48] Wang T, Sheng M, Qin Q. Sound transmission loss through metamaterial plate with lateral local resonators in the presence of external mean flow. J Acoust Soc Am, 2017, 141 (2): 1161.

[49] Van Belle L, Desmet W. Damping in a Locally Resonant Metamaterial using Inverse and Direct Unit Cell Modelling. Marseille, 2017. 